\title{
New Crystal Structure of $\mathrm{Nd}_{2} \mathrm{Ni}_{7}$ Formed on the Basis of Stacking of Block Layers
}

\author{
Yuki Iwatake ${ }^{\dagger}$, Norihiko L. Okamoto ${ }^{\dagger} *$, Kyosuke Kishida ${ }^{\dagger}$, Haruyuki Inui ${ }^{\dagger}$, Jun Ishida ${ }^{*}$ \\ Takuya Kai ${ }^{\ddagger}$ and Shigekazu Yasuokat \\ ${ }^{\dagger}$ Department of Materials Science and Engineering, Kyoto University \\ Sakyo-ku, Kyoto 606-8501, Japan \\ Technology R \& D Division, FDK Twicell Co., Ltd. \\ 307-2 Koyagi-machi, Takasaki, Gunma 370-0071, Japan
}

*Corresponding Author Contact Information:

Norihiko L. Okamoto

Department of Materials Science and Engineering, Kyoto University

Sakyo-ku, Kyoto 606-8501, Japan

Tel: $+81-75-753-5481$

Fax: +81-75-753-5461

E-mail: okamoto.norihiko.7z@kyoto-u.ac.jp 
ABSTRACT. The new crystal structure of the intermetallic compound $(\mathrm{Nd}, \mathrm{Mg})_{2}(\mathrm{Ni}, \mathrm{Al})_{7}$ has been determined by scanning transmission electron microscopy and electron diffraction. This crystal structure is different from that (either $2 H$ or $3 R$ ) usually reported for the intermetallic compound $R_{2} T_{7}$ ( $R$ : rare-earth elements and $T$ : transition-metal elements) in the constitution of block layers. While the block layer for the crystal structure usually reported is of the $R_{2} T_{7}$ stoichiometric composition consisting of one $R_{2} T_{4}$ unit layer and two $R T_{5}$ unit layers, the block layer for the new crystal structure consists of a sub-block layer of the $R T_{3}$ stoichiometry (formed with one $R_{2} T_{4}$ unit layer and one $R T_{5}$ unit layer) and a sub-block layer of the $R_{5} T_{19}$ stoichiometry (formed with one $R_{2} T_{4}$ unit layer and three $R T_{5}$ unit layers), which alternately stack on top of each other. The crystal structure is described based on the order-disorder (OD) theory and the simplest crystal structures (polytypes) among many other polytypes belonging to the same OD family is deduced to be of the $2 H$-type with the space group of $P \overline{6} m 2$ and of the $6 R$-type with the space group of $R \overline{3} m$. The experimentally observed polytype corresponds to the second simplest form of the $6 R$-type.

KEYWORDS. Hydrogen storage material, Order-disorder (OD) theory, Polytype, Scanning transmission electron microscopy, Superlattice structure, $\mathrm{La}_{2} \mathrm{Ni}_{7}$ 


\section{INTRODUCTION}

Since the discovery of the excellent hydrogen absorption-desorption properties in 2000 [1], the intermetallic compound based on $R_{2} T_{7}$ ( $R$ : rare-earth elements and $T$ : transition-metal elements) has received a considerable interest not only scientifically [2-9] but also industrially [10]. This stems from the fact that the additions of $\mathrm{Mg}$ completely alter the hydrogen absorption-desorption properties of $\mathrm{La}_{2} \mathrm{Ni}_{7}$ [1], which had been believed to exhibit properties not useful for the application as negative electrode materials of rechargeable nickel-metal hydride (Ni-MH) batteries because of the occurrence of non-rechargeable amorphization upon hydrogenation at ambient temperature [11]. Since then, many studies have devoted to the intermetallic compound based on $R_{2} T_{7}$, such as $\mathrm{La}_{2} \mathrm{Ni}_{7}$ and $(\mathrm{La}, \mathrm{Mg})_{2} \mathrm{Ni}_{7}$ in order to develop alloys that exhibit better hydrogen absorption-desorption properties as negative electrode materials of rechargeable Ni-MH batteries. One of the focuses in the alloy development has been to establish the structure-properties relationships, paying special attention to the control of phase constitution, crystal structure (polytype) by alloying additions and their preferential sites. One of the most significant scientific characteristics in the intermetallic compound based on $R_{2} T_{7}$ is in its crystal structure, as in other series compounds. The unit cells of a series of compounds in the $R-T$ system, $R T_{3}, R_{2} T_{7}$ and $R_{5} T_{19}$ are known to be made of some block layers, each consisting of one unit layer of the $R_{2} T_{4}$ (Laves)-type and some unit layers of the $R T_{5}$-type [12-14] (Fig. 1). The number $(n)$ of $R T_{5^{-}}$ type unit layers in a block layer is 1,2 and 3 for $R T_{3}, R_{2} T_{7}$ and $R_{5} T_{19}$, respectively. Thus, this series of compounds are formulated to be $R T_{x}$ with $x=(5 n+4) /(n+2)$ [12]. Of significance to note is that for all these compounds $R T_{x}$, each block layer possesses the stoichiometric composition of the corresponding intermetallic compound and that depending on the stacking of these block layers, some different polytypes (such as $2 H$ and $3 R$ according to the Ramsdell notation [15]) are generated for each of these 


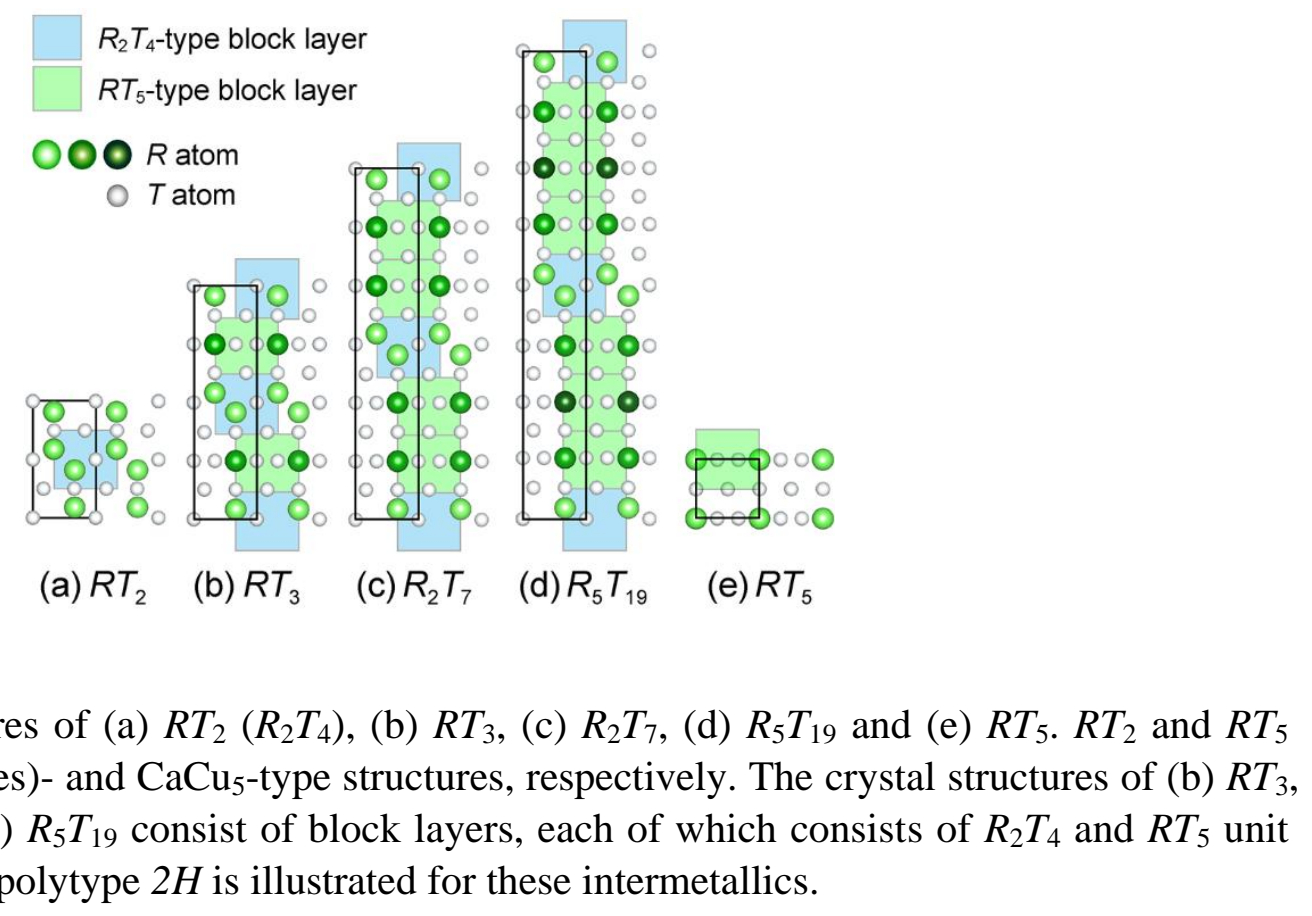

Figure 1. Crystal structures of (a) $R T_{2}\left(R_{2} T_{4}\right)$, (b) $R T_{3}$, (c) $R_{2} T_{7}$, (d) $R_{5} T_{19}$ and (e) $R T_{5} . R T_{2}$ and $R T_{5}$ have $\mathrm{C} 15$ (Laves)- and $\mathrm{CaCu}_{5}$-type structures, respectively. The crystal structures of (b) $R T_{3}$, (c) $R_{2} T_{7}$ and (d) $R_{5} T_{19}$ consist of block layers, each of which consists of $R_{2} T_{4}$ and $R T_{5}$ unit layers, and the polytype $2 \mathrm{H}$ is illustrated for these intermetallics.

compounds $R T_{x}$ (Fig. 1). During the course of research on the development of alloys based on the intermetallic compound $R_{2} T_{7}$, we have recently found a new crystal structure for the $R_{2} T_{7}$ phase in the $\mathrm{Nd}-\mathrm{Mg}-\mathrm{Ni}-\mathrm{Al}$ quaternary system, the crystal structure of which cannot simply be described according to the above-mentioned method with the stacking of block layers possessing the stoichiometric composition of the corresponding intermetallic compound.

In the present paper, we report the results of crystal structure assessment made for the $R_{2} T_{7}$ phase in the Nd-Mg-Ni-Al quaternary system by means of high-resolution scanning transmission electron microscopy (STEM) as well as electron diffraction. We describe the crystal structure based on the order-disorder (OD) theory [16-21] and deduce the simplest crystal structures (polytypes) of many other polytypes belonging to the same OD family, together with their space groups. 


\section{EXPERIMENTAL PROCEDURES}

Two different Nd-Mg-Ni-Al quaternary alloys (1 and 2) were prepared by induction-melting the constituent elements in their desired molar fractions, followed by annealing at $950{ }^{\circ} \mathrm{C}$ for $48 \mathrm{~h}$ in Ar gas and furnace-cooling. The molar ratio of $(\mathrm{Nd}+\mathrm{Mg}):(\mathrm{Ni}+\mathrm{Al})$ was set at 2:7 for both alloys, assuming all $\mathrm{Nd}$ and $\mathrm{Mg}$ atoms occupy the $R$ sites while all $\mathrm{Ni}$ and $\mathrm{Al}$ atoms occupy the $T$ sites in the intermetallic compound $R_{2} T_{7}$. The alloy 2 contains less $\mathrm{Nd}$ and more $\mathrm{Mg}$ than the alloy 1 , as tabulated in Table 1. Microstructures (phase constitution) and their crystal structures in these alloys were examined with JEM-2100F scanning transmission electron microscope (STEM) operated at $200 \mathrm{kV}$. Phase compositions were examined by energy-dispersive spectroscopy (EDS) in the STEM. Thin foils for STEM observations were perforated by ion milling with $3 \mathrm{keV}$ Ar ions.

Table 1. Nominal compositions of the alloys 1 and 2.

\begin{tabular}{ccccc}
\hline \hline (at.\%) & $\mathrm{Nd}$ & $\mathrm{Mg}$ & $\mathrm{Ni}$ & $\mathrm{Al}$ \\
\hline Alloy 1 & 16.7 & 5.6 & 74.4 & 3.3 \\
Alloy 2 & 15.6 & 6.7 & 74.4 & 3.3 \\
\hline
\end{tabular}

\section{RESULTS}

The alloy 1 consists of four phases, $R_{2} T_{7}, R_{5} T_{19}, R T_{5}$ and $R T_{2}$, with $R_{2} T_{7}$ being the major constituent phase. Figures 2(a) and (b) show a typical high-resolution HAADF (high-angle annular dark-field)-STEM image and a selected-area electron diffraction (SAED) pattern with the [2 1110$]$ incidence of a crystal grain of the $R_{2} T_{7}$ phase. EDS analysis indicates that the chemical composition of the $R_{2} T_{7}$ phase is on average Nd-2.1Mg-79.5Ni-1.4Al (at.\%). The $R_{2} T_{7}$ phase of Fig. 2(a) consists of 
block layers of the $R_{2} T_{7}$ stoichiometric composition formed with one $R_{2} T_{4}$ unit layer and two $R T_{5}$ unit layers. These block layers stack in the $c$-axis direction so as to form the AB-type stacking ( $2 H$ polytype), as schematically illustrated in the inset of Fig. 2(a). Stacking faults (the disturbance of the AB-type stacking as well as the intergrowth of other phases with different block layer structures) were observed only sporadically in grains of the $R_{2} T_{7}$ phase. The SAED pattern of Fig. 2(b) is consistently indexed as that of $R_{2} T_{7}$ of the $2 H$ polytype. The discreteness of diffraction spots together with the absence of streaks in the SAED pattern of Fig. 2(b) is consistent with the high-resolution STEM image of Fig. 2(a) showing a very regular AB-type stacking ( $2 H$ polytype) of the $R_{2} T_{7}$ phase without stacking faults.

The alloy 2 also consists of four phases, $R_{2} T_{7}, R T_{5}, R T_{3}$ and $R T_{2}$, with $R_{2} T_{7}$ being the major constituent phase. EDS analysis indicates that the chemical composition of the $R_{2} T_{7}$ phase is on average $\mathrm{Nd}-5.6 \mathrm{Mg}-77.2 \mathrm{Ni}-2.9 \mathrm{Al}$ (at.\%), which is a little more in $\mathrm{Mg}$ and $\mathrm{Al}$ than in the $R_{2} T_{7}$ phase in the alloy 1. Figures 3(a) and (b) show a typical high-resolution HAADF-STEM image and a SAED pattern with the [21 $1 \overline{1} 0]$ incidence of a crystal grain of the $R_{2} T_{7}$ phase. The SAED pattern of Fig. 3(b) is completely different from that of the $R_{2} T_{7}$ phase of the $2 H$ polytype. Analysis of the stacking of block layers in Fig. 3(b) indicates that unlike in the $R_{2} T_{7}$ phase ever reported, the block layer of this $R_{2} T_{7}$ phase consists of a block layer of the $R T_{3}$ stoichiometry (formed with one $R_{2} T_{4}$ unit layer and one $R T_{5}$ unit layer) and that of the $R_{5} T_{19}$ stoichiometry (formed with one $R T_{3}$ unit layer and three $R T_{5}$ unit layers) alternately stacked on top of each other. This new $R_{2} T_{7}$ phase is thus described to consist of the block layer of the $R_{2} T_{7}$ stoichiometry formed with alternatively stacked sub-block layers of the $R T_{3}$ and $R_{5} T_{19}$ stoichiometries. The $R_{2} T_{7}$ block layer stacks in the $c$-axis direction so as to form the ABCtype stacking to complete the 

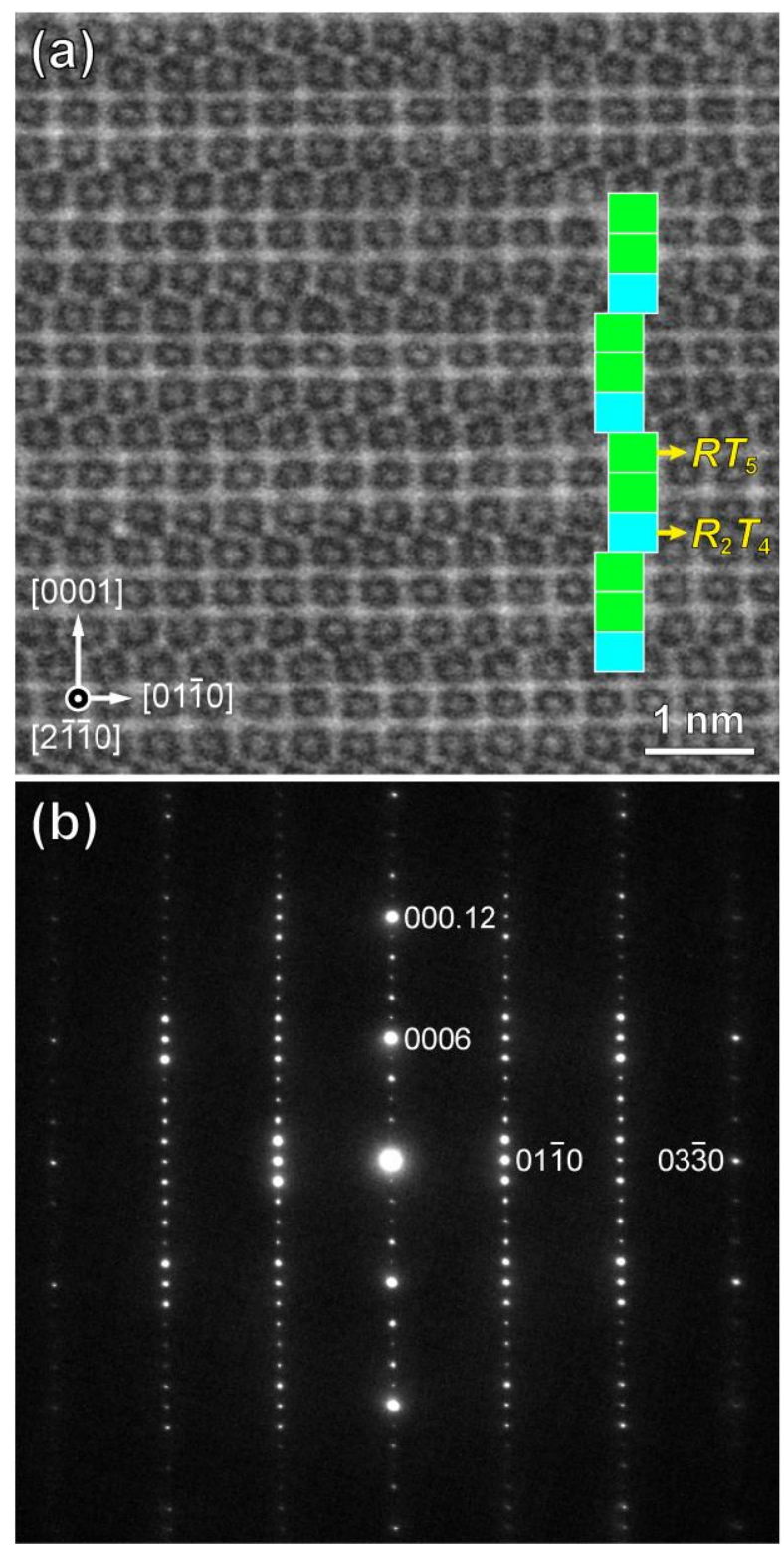

Figure 2. (a) Typical high-resolution HAADF-STEM image and (b) SAED pattern with the [2 $\overline{1} \overline{1} 0]$ incidence for a crystal grain of the $R_{2} T_{7}$ phase in the alloy 1 .
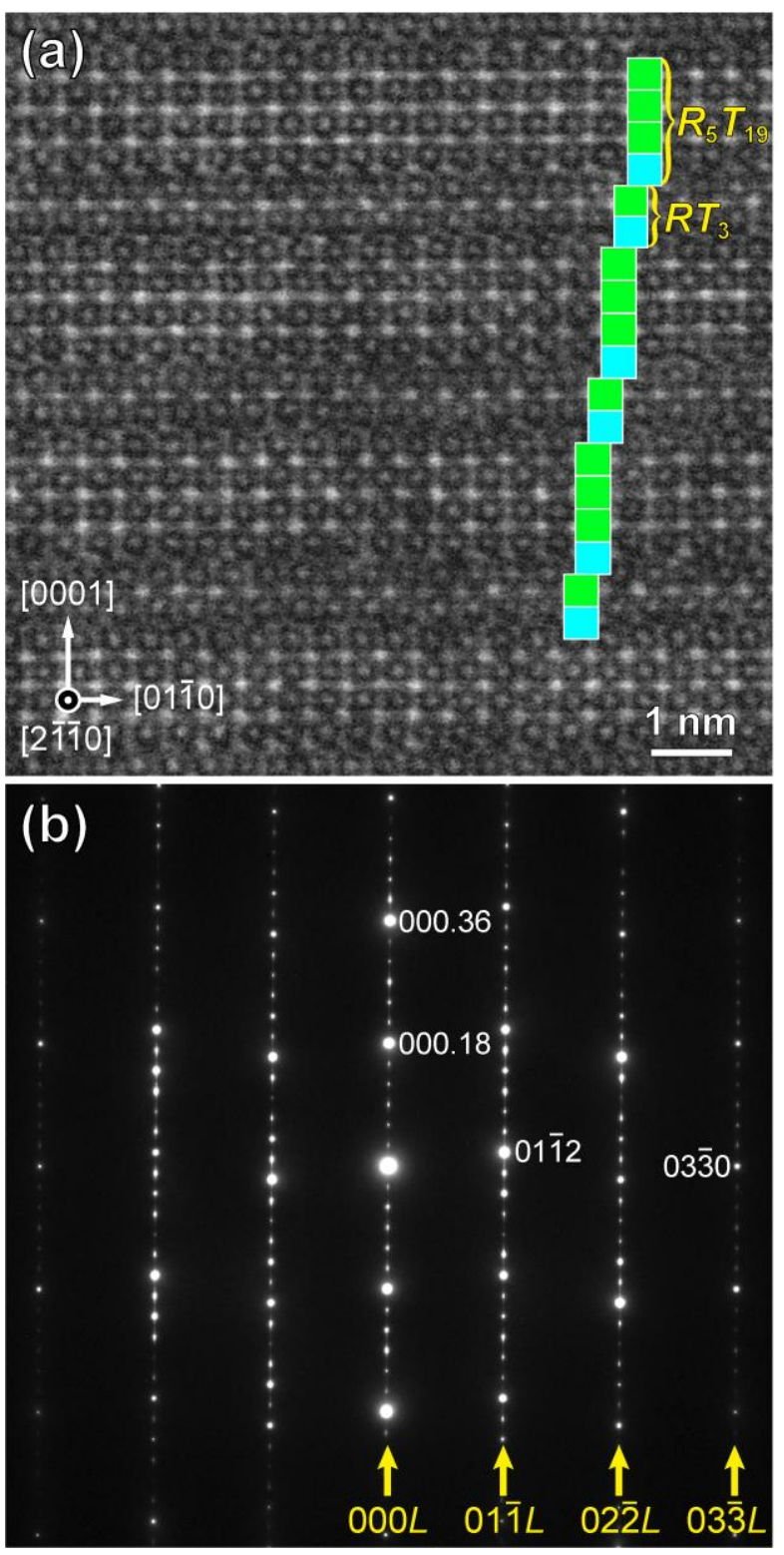

Figure 3. (a) Typical high-resolution HAADF-STEM image and (b) SAED pattern with the [2 $\overline{1} 10]$ incidence for a crystal grain of the $R_{2} T_{7}$ phase in the alloy 2 .

three-layer unit cell, as schematically shown in the inset of Fig. 3(a). The ABC-type three-layer stacking is usually observed for this $R_{2} T_{7}$ phase and seems the lowest energy configuration. The space group of $R \overline{3} m$ can thus be assigned to this new crystal structure. The SAED pattern of Fig. 
3(b) is consistently indexed with the assigned space group of $R \overline{3} m$ for the new crystal structure of the $R_{2} T_{7}$ phase. Although the crystal structure can also be considered as an intergrowth structure of the $R T_{3}$ and $R_{5} T_{19}$ phases, we believe the structure is regarded as a new crystal structure in view of the very regularity of the incidence of sub-block layers of the $R T_{3}$ and $R_{5} T_{19}$ stoichiometry and rather wide areas of the appearance of the structure, as shown in a low-magnification HAADFSTEM image of Fig. 4. Being consistent with the occurrence of streaks in the SAED pattern of Fig. 3(b), the incidence of stacking faults SF1 and SF2 are observed in Fig. 4, although the density of stacking faults is not generally remarkably high in other areas. A sub-block layer of the $R_{5} T_{19}$ stoichiometry is missing for the stacking faults SF1, while for the stacking fault SF2, a sub-block layer of the $R T_{3}$ stoichiometry is missing. Stacking fault between adjacent block layers of the $R_{2} T_{7}$ stoichiometry to disturb the ABC-type three-layer stacking of the new crystal structure is rarely observed. In the same specimen, crystal grains of the $R_{2} T_{7}$ phase with the well-known crystal

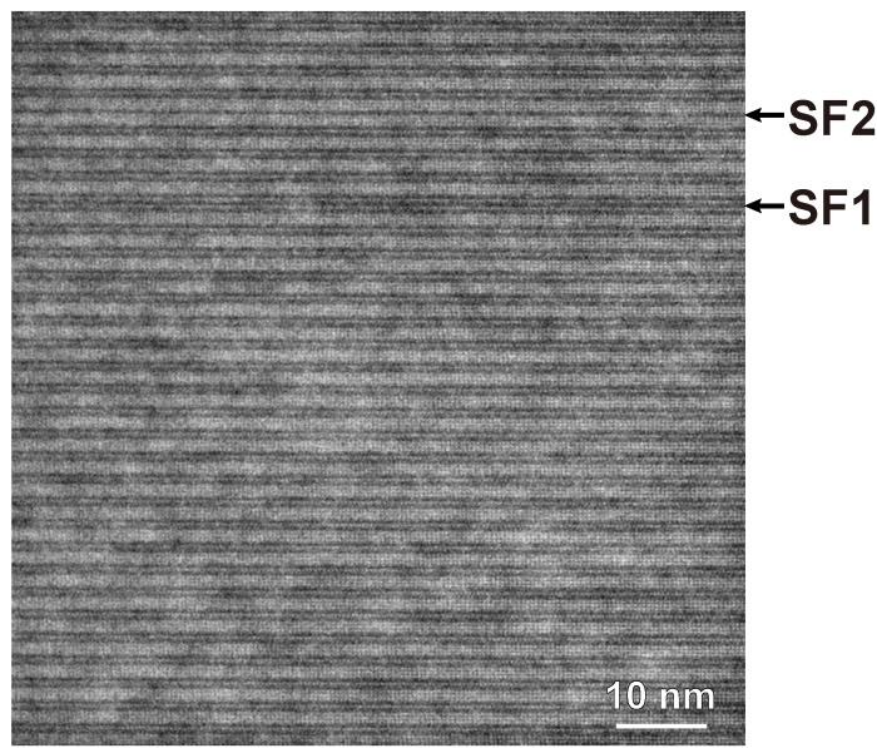

Figure 4. Low-magnification HAADF-STEM image of the $R_{2} T_{7}$ phase in the alloy 2. Positions of stacking faults (SF1 and SF2) are indicated in the right of the figure. 
structure of the $2 \mathrm{H}$ polytype (space group $P 6_{3} / \mathrm{mmc}$ ) are also frequently observed. These grains tend to have chemical compositions with less $\mathrm{Mg}$ and $\mathrm{Al}$ than grains of the $R_{2} T_{7}$ phase with the new crystal structure, although the difference in chemical composition is not always remarkable.

\section{DISCUSSION}

4.1. The crystal structure description of $R T_{3}, R_{2} T_{7}$ and $R_{5} T_{19}$ with the order-disorder theory

Although two polytypes, $2 H$ and $3 R$ are frequently described to be observed experimentally for a series of compounds in the $R-T$ system, $R T_{3}, R_{2} T_{7}$ and $R_{5} T_{19}$, many other polytypes with larger unit cell dimensions along the stacking direction as well as virtually onedimensionally stacking disordered structures are possible to be formed, since their unit cell is based on the stacking of block layers, each of which consists of one unit layer of the $R T_{2}$ (Laves)type and some unit layers of the $R T_{5}$-type. Here, we describe the crystal structures of a series of compounds in the $R$-T system $\left(R T_{3}, R_{2} T_{7}\right.$ and $\left.R_{5} T_{19}\right)$ according to the so-called order-disorder theory [16-21] in order to generalize the crystal structures. The theory of the OD structure (OD theory) was originally developed to describe crystal structures with one-dimensional stacking disorder observed in many minerals, such as wollastonite [22-27]. In the OD theory, a crystal structure is described with two sets of partial operations (POs) of symmetry, $\lambda$-POs and $\sigma$-POs [16-21]. The $\lambda$-POs correspond to POs that transform an OD layer into itself, while $\sigma$-PO transforms an OD layer into an adjacent one above it. For a given set of $\lambda$-POs, i.e. a given layer group, a set of $\sigma$-POs can be derived based on the symmetry of the layer group. The POs sustaining the orientation of the layer are denoted as $\tau$-POs, while those turning the layer upside down are called $\rho$-POs. Both $\lambda$ - and $\sigma$ - POs can be either $\tau$ or $\rho$. A whole family of the derivative structures described with a complete set of POs is called an OD-groupoid family. 
The choice of OD layers in the crystal structure description according to the OD theory is not unique and there are some different possibilities. The block layer with the stoichiometric composition (either $R T_{3}, R_{2} T_{7}$ or $R_{5} T_{19}$ ) is one of them. The structural block can indeed be divided into two different OD layers corresponding respectively to sub-block layers of the $R_{2} T_{4}$ (Laves)and the $R T_{5}$-types, as shown in Figs. 5(a)-(c). This choice of OD layers is convenient to describe the crystal structures of these three intermetallic compounds in a quite similar way according to the OD theory. These two different OD layers are denoted in the following as $L_{n}$ and $M_{n}$ :

(i) OD layer $L_{n}$, corresponding to a sub-block layer of the $R_{2} T_{4}$-type (Figs. 5(a)-(c) and 6(a)-(b)). The layer group of the OD layer $L_{n}$ is $p \overline{3} m 1$ (short symbol) or $\operatorname{Pmmm}(\overline{3}) 11$ (full seven-entry symbol). The layer is non-polar. There is one $\lambda$ - $\rho$ plane.

(ii) OD layer $M_{n}$, corresponding to a sub-block layer of the $R T_{5}$-type (Figs. 5 (a)-(c)). This OD layer contains all unit layers of the $R T_{5}$-type in the sub-block layer for each of these three compounds. Thus, the number of unit layers of the $R T_{5}$-type contained in the OD layer is 1,2 and 3 for $R T_{3}, R_{2} T_{7}$ and $R_{5} T_{19}$, respectively. The number of unit layers of the $R T_{5}$-type contained in the OD layer does not alter the symmetry of the OD layer. The layer group of the OD layer $M_{n}$ is $p 6 / \mathrm{mmm}$ (short symbol) or Pmmm(6/m) mmm (full seven-entry symbol). The layer is non-polar. There is one $\lambda-\rho$ plane.

The crystal structures of these three intermetallic compounds can thus be considered to consist of these two OD layers stacked alternately along the $c$ direction. The diagrams of symmetry elements for the $p \overline{3} \mathrm{~m} 1$ and $p 6 / \mathrm{mmm}$ layer groups corresponding to the $\lambda$-POs in the OD layers $L_{n}$ and $M_{n}$ are illustrated in Figs. 6(g) and (h), respectively [28]. Because the crystal structure is composed of two OD layers both of which are non-polar (as is evident from the presence of $\lambda$ - $\rho$ plane), all the three intermetallics can be considered to belong to the category IV OD structures [20, 29-31]. The 
(a) $2 \mathrm{H}-\mathrm{R} T_{3}(n=1)$

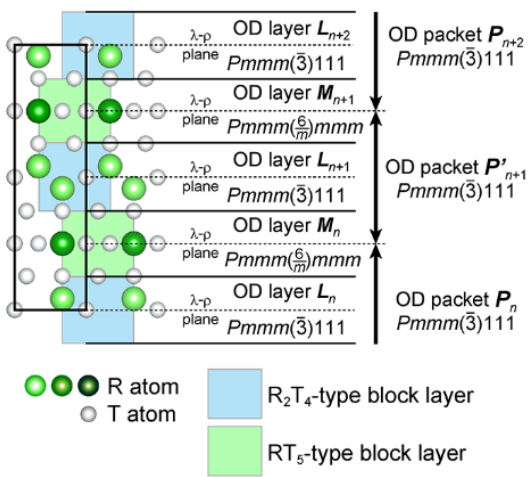

(b) $2 \mathrm{H}-\mathrm{R}_{2} \mathrm{~T}_{7}(n=2)$

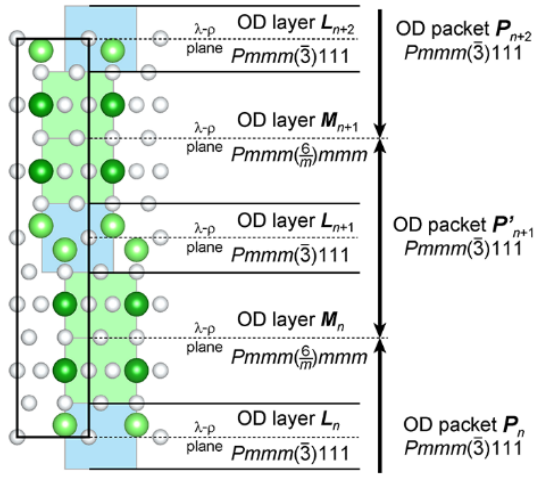

(c) $2 H-R_{5} T_{19}(n=3)$

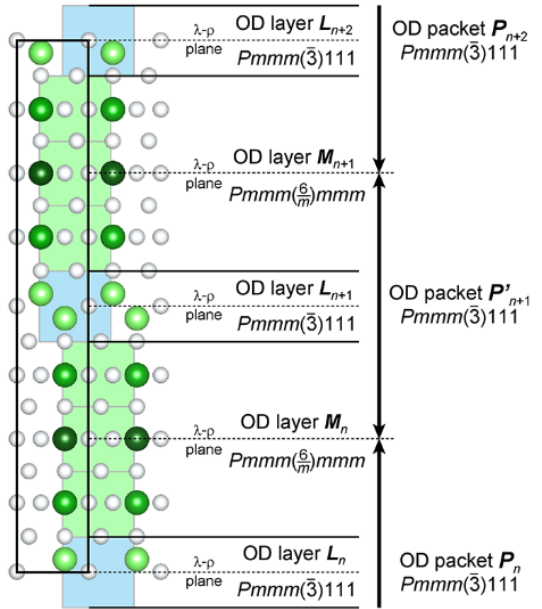

Figure 5. Crystal structures of (a) $2 H-R T_{3}$, (b) $2 H-R_{2} T_{7}$, and (c) $2 H-R_{5} T_{19}$ projected along the [2 $\overline{1}$ $10]$ direction. The constituting OD layers and packets are described in the right-hand of each figure.

choice of OD layers in OD structures of the category IV is not unique and a boundary between two adjacent OD layers can be set in more than one way [20]. Here, the boundary between two adjacent $L_{n}$ and $M_{n}$ OD layers is set so that $T$ atoms on the boundary (highlighted with light blue color in Fig. 6) belong to both OD layers.

The so-called $N F Z$ relation $[18,20,21]$ is useful to consider the number of geometrically equivalent stacking positions of the OD layers. The $N F Z$ relation calculates the value of $Z$, the number of the possible distinct positions of the OD layer $L_{p+1}$ relative to the fixed position of the OD layer $L_{p}$. The value of $Z$ depends on the $\operatorname{order} N$, the general multiplicity of the group of the $\lambda$ $\tau$-POs valid for the single OD layer $L_{p}$, as well as on the order $F$, the general multiplicity of the group of the $\lambda$ - $\tau$-POs valid both for the single OD layer $L_{p}$ and for the pair of the adjacent OD layers $L_{p}$ and $L_{p+1}$. For OD structures in the category IV, the value of $Z$ is calculated through the relation, $Z=N / F$. In the case for stacking an OD layer $M_{n}$ on top of an OD layer $L_{n}$, the order $N$ 
for the OD layer $L_{n}$ is six out of 12 symmetry operations of the $p \overline{3} m 1$ layer group [28]. Since all the six operations are valid for the pair of the adjacent OD layers $L_{n}$ and $M_{n}$, the order $F$ is also six. Thus, the number $Z=N / F=6 / 6=1$, indicating that there is only one possible position for the OD layer $M_{n}$ to stack on top of the OD layer $L_{n}$. In the case for stacking an OD layer $L_{n+1}$ on top of that an OD layer $M_{n}$, on the other hand, the order $N$ for the OD layer $M_{n}$ is 12 out of 24 symmetry operations of the $\mathrm{p6} / \mathrm{mmm}$ layer group [28]. Since only six out of the 12 operations are valid for the pair of the adjacent OD layers $M_{n}$ and $L_{n+1}$, the order $F$ is also six. Thus, the number $Z=N / F=$ $12 / 6=2$. This indicates that there are two possible stacking positions when stacking an OD layer $L_{n+1}$ on top of that an OD layer $M_{n}$.

The symmetry of an OD structure is not described by a space group but is by a groupoid, which is a set of $\lambda$ - and $\sigma$-POs [16-21]. A whole family of derivative structures described with the

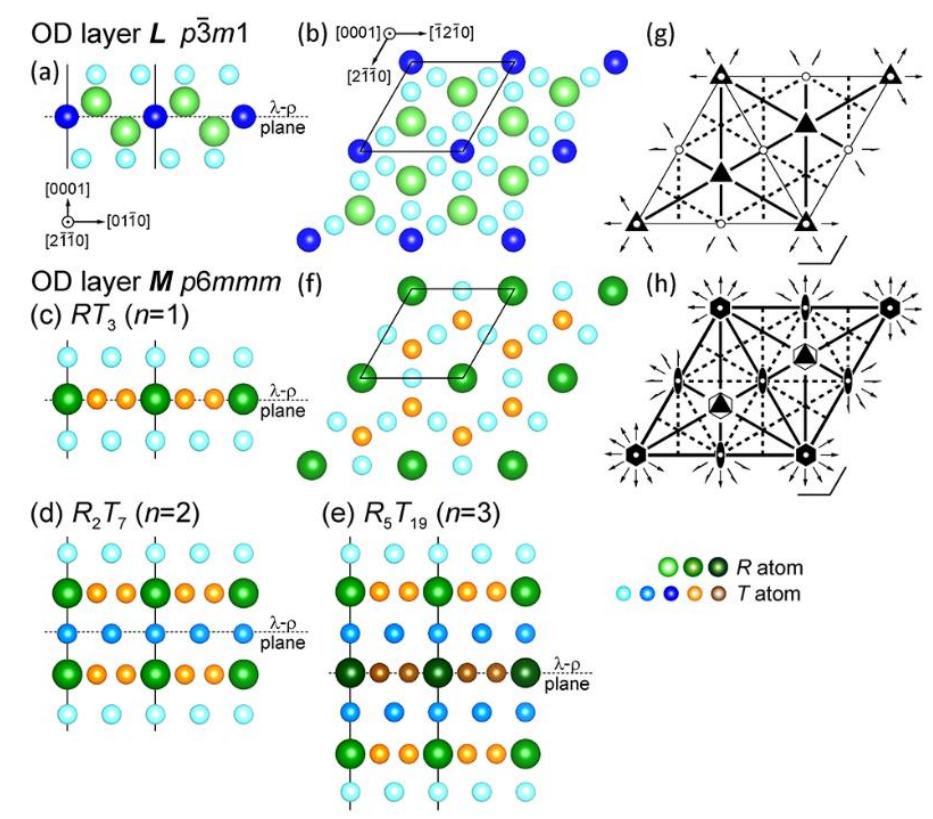

Figure 6. (a,c,d,e) [2 $\overline{1} \overline{1} 0]$ and (b,f) [0001] projections of the OD layers (a,b) $L$ and (c,d,e) $M$, respectively, in the (c) $R T_{3}$, (d) $R_{2} T_{7}$, and (e) $R_{5} T_{19}$ phases. (g,h) Diagrams of symmetry elements for the $p \overline{3} \mathrm{~m} 1$ and $p 6 / \mathrm{mmm}$ layer groups corresponding to the $\lambda$-POs in the OD layers $L$ and $M$, respectively. 
same set of $\lambda$ - and $\sigma$-POs is called an OD groupoid family and is generally described with the socalled OD-groupoid family symbol, which consists of two lines [16-21]. For the OD structures in the category IV composed of two different kinds of OD layers, the first line presents the $\lambda$-POs of the layer groups of the constituting OD layers, while the second line presents the positional relation between the adjacent OD layers, given in a square bracket by two components of the vector connecting the origins of the two adjacent layers projected into the layer plane [20, 21, 2931]. The OD groupoid family symbol for all three intermetallics is thus described as,

$$
\left.\operatorname{Pmmm}\left(\frac{6}{m}\right) \text { mmm Pmmm( } \operatorname{3}\right) 111
$$

$[2 / 3,1 / 3]$

Of significance to note is that the OD groupoid family symbol is the same for all three intermetallics and does not alter with the thickness (the number of unit layers of the $R T_{5}$-type) of the OD layer $M_{n}$.

It is often more instructive and useful to describe OD structures with structural units called OD packets, which by definition represent the smallest continuous part of the OD structure with the stoichiometric composition and is larger than individual OD layers considered previously [32]. The OD packet consists of half the OD layer $M_{n-1}$, the whole OD layer $L_{n}$ and half the OD layer $M_{n}$ with the packet boundaries being taken to coincide with the $\lambda-\rho$ plane of the OD layers $M_{n-1}$ and $M_{n}$ (Figs. 5 and 7(a)). The OD packet $P_{n}$ is thus virtually identical with the structural blocks used for explaining the crystal structures in Section 1, although the packet boundary is shifted by half the OD layer $M_{n}$ when compared with that between structural blocks. Here, the boundary between two adjacent OD packets is set so that $R$ and $T$ atoms on the $\lambda$ - $\rho$ plane in the OD layer $M_{n}$ belong to both OD packets (highlighted with green and orange color in Fig. 6(c), cyan color in Fig. 

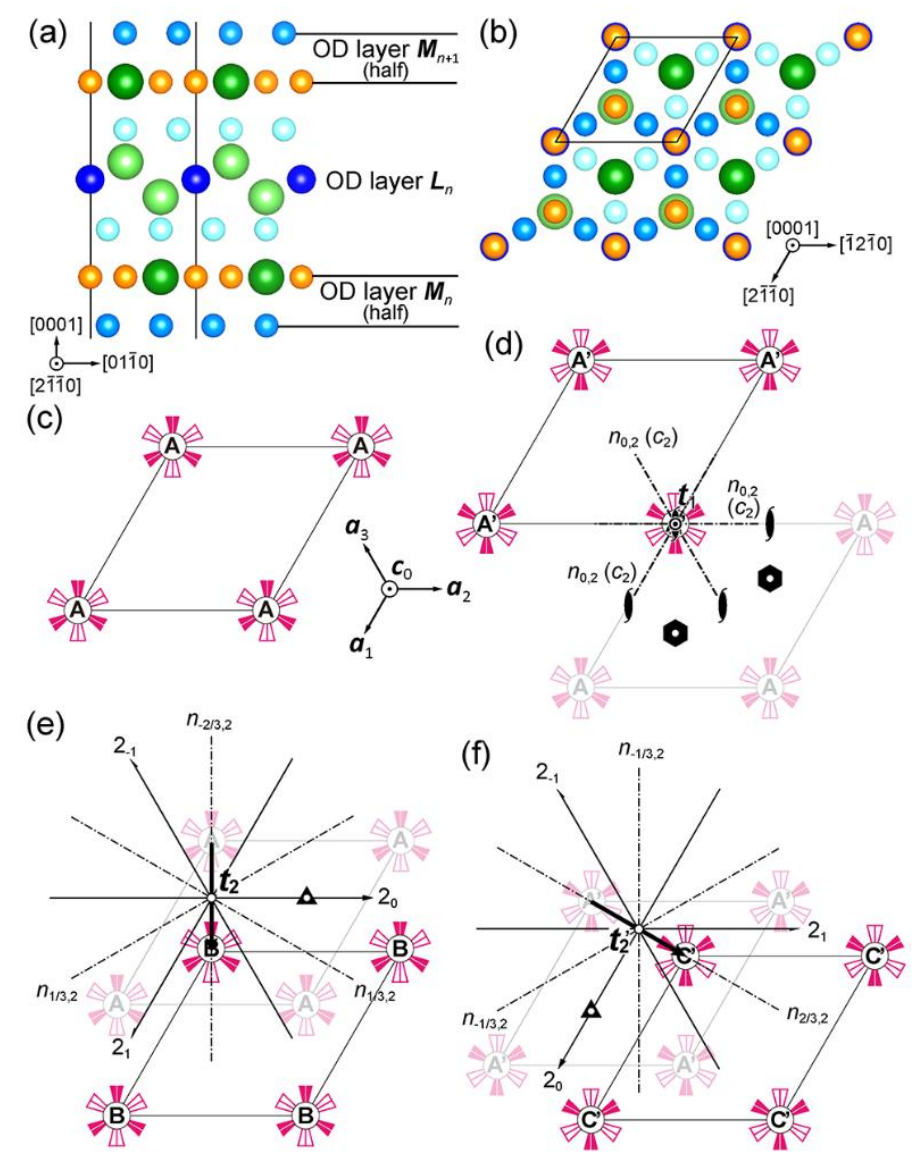

Figure 7. (a) [2 $\overline{1} \overline{1} 0]$ and (b) [0001] projections of the OD packet in the $R_{2} T_{7}$ phase. (c) Arrays of symbolic figures showing the symmetry of the OD packet. (d,e) Two different manners of stacking the adjacent OD packet on the preceding OD packet at the position A. (d) $A \Rightarrow A^{\prime}$ with the stacking vector $\boldsymbol{t}_{1}$, (e) $\mathrm{A} \Rightarrow \mathrm{B}$ with the stacking vector $\boldsymbol{t}_{2}$. (f) One of the two manners of stacking the adjacent OD packet on the preceding OD packet at the position $A^{\prime}\left(A^{\prime} \Rightarrow C^{\prime}\right)$.

6(d), and dark green and brown color in Fig. 6(e), respectively). The symmetry of the OD packet for all three intermetallics is identical with the symmetry of the OD layer $L_{n}, p \overline{3} m 1$. It is useful to further describe the OD packet with a symbolic figure, which simply illustrates the symmetry of the OD packet [33]. The OD packet projected along the stacking direction and symbolic figure for the OD packet are shown in Figs. 7(b) and (c), respectively. The symbolic figure has $\overline{3}$ symmetry about the stacking direction and mirror planes perpendicular to the $\langle 11 \overline{2} 0>$ directions. The origin 
of the adjacent packet $P_{n+1}$ may be shifted relative to that of the packet $P_{n}$ by either of the following stacking vectors,

$$
\begin{aligned}
& \boldsymbol{t}_{1}=\boldsymbol{c}_{0} \\
& \boldsymbol{t}_{2}=\frac{2}{3} \boldsymbol{a}_{1}+\frac{1}{3} \boldsymbol{a}_{2}+\boldsymbol{c}_{0}
\end{aligned}
$$

where $c_{0}$ correponds to the unit vector of the OD packet along the stacking direction. In the former case, the packet $P_{n+1}$ is stacked right above the packet $P_{n}$ after \pm 60 or $180^{\circ}$ rotation about the $c$ axis $\left(\mathrm{A} \rightarrow \mathrm{A}^{\prime} \text { : Fig. } 7(\mathrm{~d})\right)^{1}$. In the latter case, the packet $P_{n+1}$ is stacked obliquely above the packet $P_{n}$ without rotation $(\mathrm{A} \rightarrow \mathrm{B}$ : Fig. $7(\mathrm{e}))$. When the packet $P_{n}$ has a different orientation $( \pm 60$ or $180^{\circ}$ rotation about the $c$-axis; A' position), the packet $P_{n+1}$ can be stacked right above the packet $P_{n}$ after \pm 60 or $180^{\circ}$ rotation about the $c$-axis $\left(\mathrm{A}^{\prime} \rightarrow \mathrm{A}\right)$, after shifting the origin by the following stacking vector,

$$
\boldsymbol{t}_{1}^{\prime}=\boldsymbol{c}_{0}
$$

or obliquely above the packet $P_{n}$ without rotation $\left(\mathrm{A}^{\prime} \rightarrow \mathrm{C}\right.$ : Fig. 7(f)) after shifting the origin by the following stacking vector,

$$
\boldsymbol{t}_{2}^{\prime}=\frac{1}{3} \boldsymbol{a}_{1}+\frac{2}{3} \boldsymbol{a}_{2}+c
$$

The stacking vector $\boldsymbol{t}_{1}$ shifts the stacking positions like $\mathrm{A} \rightarrow \mathrm{A}^{\prime}, \mathrm{B} \rightarrow \mathrm{B}^{\prime}, \mathrm{C} \rightarrow \mathrm{C}^{\prime}$, while $\boldsymbol{t}_{2}$ and $\boldsymbol{t}_{2}{ }_{2}$ shift the stacking positions like $\mathrm{A} \rightarrow \mathrm{B}, \mathrm{B} \rightarrow \mathrm{C}, \mathrm{C} \rightarrow \mathrm{A}$, and $\mathrm{A}^{\prime} \rightarrow \mathrm{C}^{\prime}, \mathrm{B}^{\prime} \rightarrow \mathrm{A}^{\prime}, \mathrm{C}^{\prime} \rightarrow \mathrm{B}^{\prime}$,

\footnotetext{
${ }^{1}$ The apostrophe means that the OD packet has the opposite orientation within the basal plane.
} 
respectively. An infinite number of periodic and non-periodic polytypes can thus be obtained for these three intermetallics $\left(R T_{3}, R_{2} T_{7}\right.$ and $\left.R_{5} T_{19}\right)$ by a random occurrence of the stacking vectors $t_{i}$ and $\boldsymbol{t}_{i}^{\prime}(i=1$ and 2$)$.

Among the periodic polytypes, the simplest ones are called maximum degree of order (MDO) polytypes. Each MDO polytype is characterized by a generating operation, whose continuous application gives rise to the MDO structure. In the present case of the OD groupoid family for $R T_{x}$ with $x=(5 n+4) /(n+2)$, one generating operation is the $\sigma$-PO of $n_{0,2}\left(c_{2}\right)$, indicated in Fig. 7(d). Continuous application of this operation yields a MDO polytype (MDO1) with the AA' stacking sequence. The unit cell of the MDO1 polytype belongs to the space group of $P 6_{3} / m m c$ with a hexagonal unit cell of $\boldsymbol{a}_{1}^{(M D O 1)}=\boldsymbol{a}_{1}, \boldsymbol{a}_{2}^{(M D O 1)}=\boldsymbol{a}_{2}$ and $\boldsymbol{c}^{(\mathrm{MDO} 1)}=2 \boldsymbol{c}_{0}$ because the $\sigma$-PO of $n_{0,2}$ $\left(c_{2}\right)$ (Fig. 7(d)) becomes a total operation, $c$ glide plane, in the hexagonal cell. In the Ramsdell notation, the MDO1 polytype is designated $2 \mathrm{H}$. Another generating operation is the $\sigma-\mathrm{PO}$ of a glide plane of $n_{1 / 3,2}$, indicated in Fig. 7(e). Continuous application of this operation results in another MDO polytype (MDO2) with the ABC stacking sequence. The MDO2 polytype belongs to the space group of $R \overline{3} m$ with the rhombohedral unit cell of $\boldsymbol{a}_{1}^{(M D O 2)}=\boldsymbol{a}_{1}, \boldsymbol{a}_{2}^{(M D O 2)}=\boldsymbol{a}_{2}$ and $\boldsymbol{c}^{(\mathrm{MDO} 2)}=3 \boldsymbol{c}_{0}$ because the $\sigma-\mathrm{PO}$ of $n_{1 / 3,2}$ becomes a total operation, $g(1 / 6,1 / 3,1 / 3)$, in the rhombohedral cell. In the Ramsdell notation, the MDO2 polytype is designated $3 R$.

4.2. The crystal structure description of $R_{2} T_{7}$ of the new crystal structure with the order-disorder theory

We now describe the new crystal structure of $R_{2} T_{7}$ we identified presently with the OD theory. Although it is possible to choose OD layers as in the previous section (Figs. 5, 6, and 7), 
the crystal structure description is rather complicated because the structural block with the $R_{2} T_{7}$ stoichiometric composition consists of as many as three different kinds of OD layers $\left(L_{4 n}, M_{4 n+1}\right.$, $L_{4 n+2}$ and $M^{\prime \prime \prime}{ }_{4 n+3}$, with $M_{4 n+1}$ and $M^{\prime \prime \prime}{ }_{4 n+3}$ containing one and three unit layers of the $R T_{5}$-type, respectively). To simplify the description, two different kinds of OD layers can be chosen as follows.

(i) OD layer $L^{\prime}\left(L_{3 n}^{\prime}\right.$ and $\left.L_{3 n+1}^{\prime}\right)$, consisting of a $R_{2} T_{4}$-type unit layer sandwiched by half the $R T_{5^{-}}$ type unit layer on both sides (top and bottom) (Fig. 8 and Figs. 9(a), (b)). The layer group of the OD layer $L^{\prime}$ is $p \overline{3} m 1$ (short symbol) or $\operatorname{Pmmm}(\overline{3}) 111$ (full seven-entry symbol). The layer is non-polar. There is one $\lambda-\rho$ plane.

(ii) OD layer $M^{\prime}\left(M_{3 n+2}^{\prime}\right)$, consisting of a $R T_{5}$-type unit layer sandwiched by half the $R T_{5}$-type unit layer on both sides (top and bottom), i.e., two $R T_{5}$-type unit layers (Fig. 8 and Figs. 9(c), (d)). The layer group of the OD layer $M_{3 n+2}^{\prime}$ is $p 6 / \mathrm{mmm}$ (short symbol) or Pmmm(6/m)mmm (full seven-entry symbol). The layer is non-polar. There is one $\lambda$ - $\rho$ plane.

Then, the structural block with the $R_{2} T_{7}$ stoichiometric composition can simply be described to consist of OD layers $L_{3 n}^{\prime}, L_{3 n+1}^{\prime}$ and $M_{3 n+2}^{\prime}$. In the case for stacking an OD layer $L_{3 n}^{\prime}$ on top of an OD layer $M_{3 n-1}^{\prime}$, the order $N$ for the OD layer $M$ is 12 out of 24 symmetry operations of the $\mathrm{p} 6 / \mathrm{mmm}$ layer group [28]. Since only six out of the twelve operations are valid for the pair of the adjacent OD layers $M_{3 n-1}^{\prime}$ and $L_{3 n}^{\prime}$, the order $F$ is also six. Thus, the number $Z=N / F=12 / 6=$ 2 , indicating that the existence of two possible stacking positions for the OD layer $L_{3 n}^{\prime}$ to stack on top of the OD layer $M_{3 n-1}^{\prime}$. In the case for stacking an OD layer $L_{3 n+1}^{\prime}$ on top of an OD layer $L_{3 n}^{\prime}$, the order $N$ for the OD layer $L_{3 n}^{\prime}$ is six out of 12 symmetry operations of the $p \overline{3} m 1$ layer group [28]. Since only three out of the six operations are valid for the pair of the adjacent OD layers $L_{3 n}^{\prime}$, and $L_{3 n+1}^{\prime}$, the order $F$ is also three. Thus, the number $Z=N / F=6 / 3=2$, indicating that the 
existence

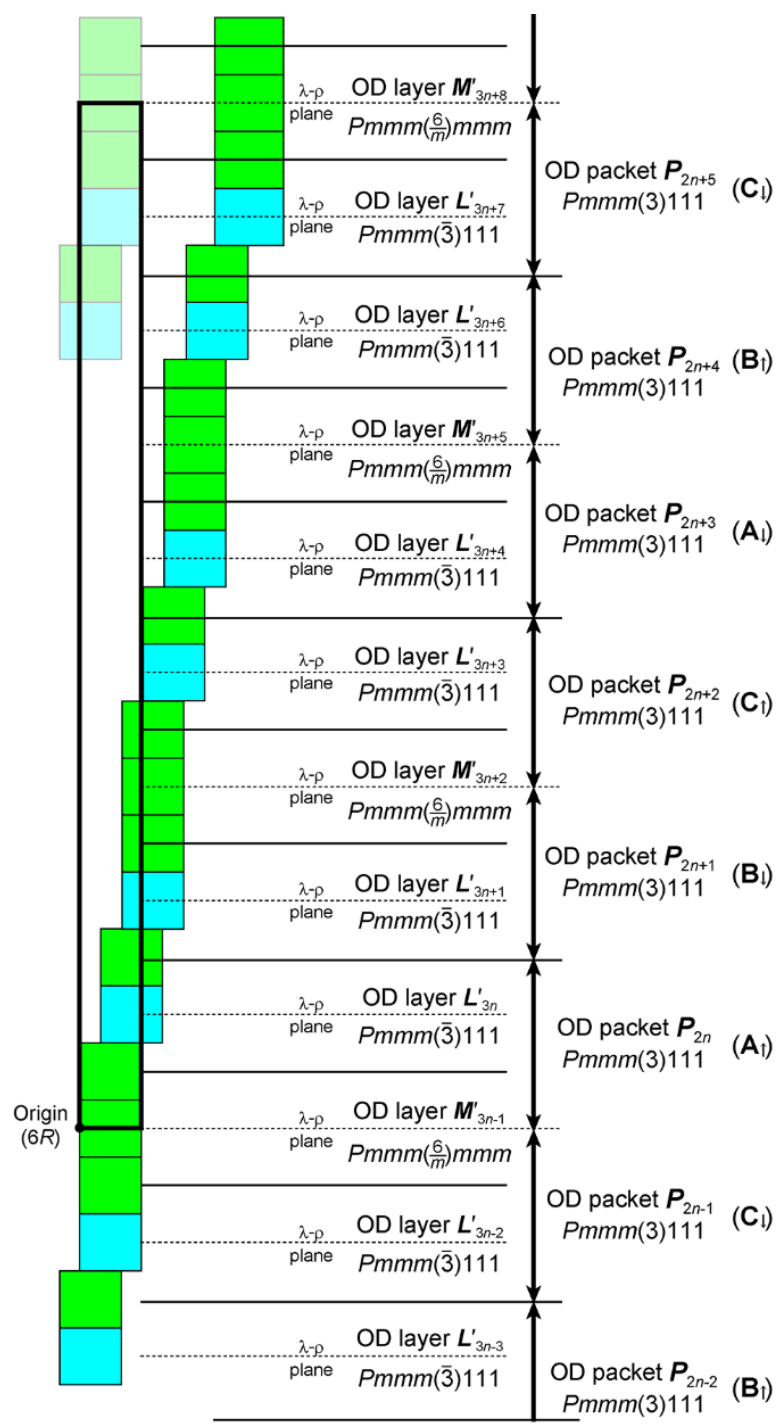

Figure 8. Schematic illustration of the crystal structure of the intermetallic compound $(\mathrm{Nd}, \mathrm{Mg})_{2}(\mathrm{Ni}, \mathrm{Al})_{7}$ (6R-polytype). The constituting OD layers and packets are described in the right-hand of the figure.
OD layer $L^{\prime} \quad p \overline{3} m 1$

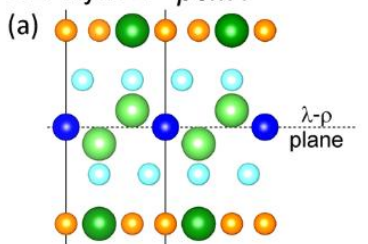

OD layer $\boldsymbol{M}^{\prime} \quad$ p6mmm

(c) 000000

$\circ \circ 00$ $00000.00_{\text {plane }}^{\lambda-p}$

$\circ \circ \circ \circ \circ$ 0000000

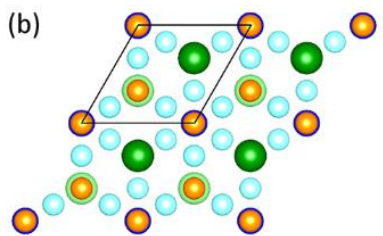

(d)

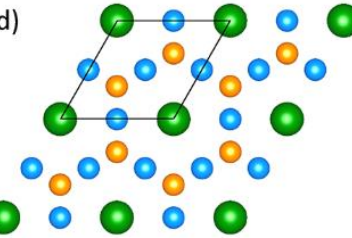

Figure 9. (a,c) [2 $\overline{1} \overline{1} 0]$ and $(b, d)$ [0001] projections of the OD layers $(\mathrm{a}, \mathrm{b}) L^{\prime}$ and $(\mathrm{c}, \mathrm{d})$ $M^{\prime}$, respectively, in the $(\mathrm{Nd}, \mathrm{Mg})_{2}(\mathrm{Ni}, \mathrm{Al})_{7}$ phase of the new crystal structure.

(a)

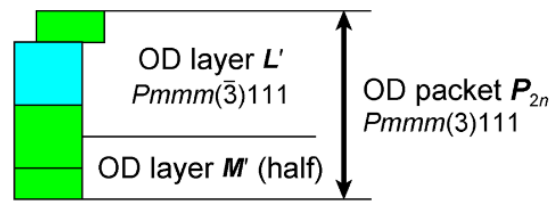

(b)

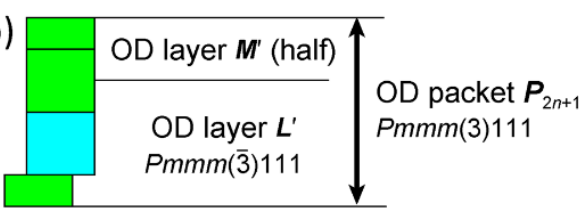

Figure 10. Schematic illustration of the OD packets $P_{2 n}$ and $P_{2 n+1}$ constituting the $(\mathrm{Nd}, \mathrm{Mg})_{2}(\mathrm{Ni}, \mathrm{Al})_{7}$ phase of the new crystal structure.

of two possible stacking positions for the OD layer $L_{3 n+1}^{\prime}$ to stack on top of the OD layer $L_{3 n}^{\prime}$. In the case for stacking an OD layer $M_{3 n+2}^{\prime}$ on top of an OD layer $L_{3 n+1}^{\prime}$, on the other hand, the order $N$ for the OD layer $L_{3 n+1}^{\prime}$ is six out of 12 symmetry operations of the $p \overline{3} m 1$ layer group. Since all 
the six operations are valid for the pair of the adjacent OD layers $L_{3 n+1}^{\prime}$ and $M_{3 n+2}^{\prime}$, the order $F$ is also six. Thus, the number $Z=N / F=6 / 6=1$, indicating that that these is only one possible position for the OD layer $M_{3 n+2}^{\prime}$ to stack on top of the OD layer $L_{3 n+1}^{\prime}$. Because the crystal structure is composed of three OD layers all of which are non-polar (as is evident from the presence of $\lambda$ - $\rho$ plane), the structure can be considered to belong again to the category IV OD structures [20, 29-31]. The OD groupoid family symbol for the crystal structure is thus described as,

$\operatorname{Pmmm}\left(\frac{6}{m}\right) m m m \operatorname{Pmmm}(\overline{3}) 111 \operatorname{Pmmm}(\overline{3}) 111$

$$
[2 / 3,1 / 3] \quad[2 / 3,1 / 3]
$$

The OD packet can be taken to consist of half the OD layer $M^{\prime}$ and the OD layer $L^{\prime}$ (Fig. 10), so that the structure can be described with the alternate stacking of the OD packet $P_{2 n}$ and $P_{2 n+1}$, which are related with each other by inversion symmetry (with respect to the origin of the OD layer $M^{\prime}$ ). The boundary between two adjacent OD packets is set so that $R$ and $T$ atoms in the OD layer $L^{\prime}$ highlighted with green and orange color in Fig. 11(a) and those in the OD layer $M^{\prime}$ highlighted with dark green and brown colors in Fig. 11(a) belong to both OD packets. The OD packet projected along the stacking direction and symbolic figure for the OD packet are shown in Figs. 11(b) and (c), respectively.

The origin of the adjacent packet $P_{2 n+1}$ may be shifted relative to that of the packet $P_{2 n}$ by either of the following stacking vectors,

$$
\begin{aligned}
& \boldsymbol{t}_{1}=\boldsymbol{c}_{0} \\
& \boldsymbol{t}_{2}=\frac{2}{3} \boldsymbol{a}_{1}+\frac{1}{3} \boldsymbol{a}_{2}+\boldsymbol{c}_{0}
\end{aligned}
$$


where $\boldsymbol{c}_{0}$ correponds to the unit vector of the OD packet along the stacking direction. In the former case, the packet $P_{2 n+1}$ is stacked right above the packet $P_{2 n}$ after \pm 60 or $180^{\circ}$ rotation about the $c$-axis $\left(\mathrm{A}_{\uparrow} \rightarrow \mathrm{A}_{\downarrow}^{\prime} \text { : Fig. 11(d) }\right)^{2}$. In the latter case, the packet $P_{2 n+1}$ is stacked obliquely
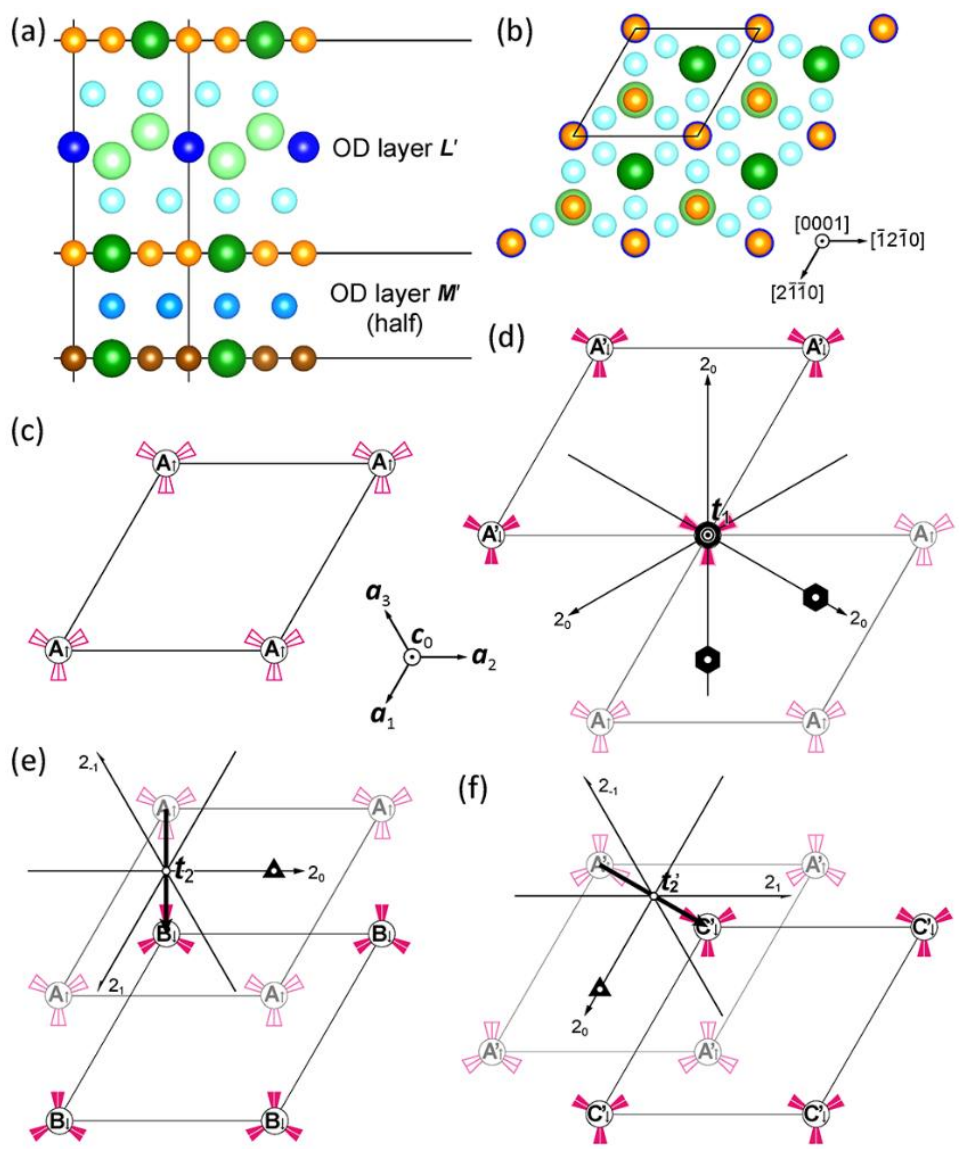

Figure 11. (a) [2 $1 \overline{1} 0]$ and (b) [0001] projections of the $\mathrm{OD}$ packet in the $(\mathrm{Nd}, \mathrm{Mg})_{2}(\mathrm{Ni}, \mathrm{Al})_{7}$ phase of the new crystal structure. (c) Arrays of symbolic figures showing the symmetry of the OD packet. (d,e) Two different manners of stacking the adjacent OD packet on the preceding OD packet at the position $A_{\uparrow}$. (d) $A_{\uparrow} \rightarrow A_{\downarrow}^{\prime}$ with the stacking vector $\boldsymbol{t}_{1}$, (e) $\mathrm{A}_{\uparrow} \rightarrow \mathrm{B}_{\downarrow}$ with the stacking vector $\boldsymbol{t}_{2}$. (f) One of the two manners of stacking the adjacent OD packet on the preceding OD packet at the position $\mathrm{A}_{\uparrow}^{\prime}\left(\mathrm{A}_{\uparrow}^{\prime} \rightarrow \mathrm{C}_{\downarrow}^{\prime}\right)$.

\footnotetext{
${ }^{2}$ The upward and downward arrows indicate that the OD layer $L$ ' is located on the top or in the bottom of the OD packet, respectively (see Figure 10).
} 
above the packet $P_{2 n}$ without rotation $\left(\mathrm{A}_{\uparrow} \rightarrow \mathrm{B}_{\downarrow}\right.$ : Fig. 11(e)). When the packet $P_{2 n}$ has a different orientation ( \pm 60 or $180^{\circ}$ rotation about the $c$-axis; $\mathrm{A}_{\uparrow}^{\prime}$ position), the packet $P_{2 n+1}$ can be stacked right above the packet $P_{2 n}$ after \pm 60 or $180^{\circ}$ rotation about the $c$-axis $\left(\mathrm{A}_{\uparrow}^{\prime} \rightarrow \mathrm{A}_{\downarrow}\right)$, after shifting the origin by the following stacking vector,

$$
\boldsymbol{t}^{\prime}=\boldsymbol{c}_{0}
$$

or obliquely above the packet $P_{n}$ without rotation $\left(\mathrm{A}_{\uparrow}^{\prime} \rightarrow \mathrm{C}_{\downarrow}^{\prime}\right.$ : Fig. 11(f)) after shifting the origin by the following stacking vector,

$$
t_{2}^{\prime}=\frac{1}{3} a_{1}+\frac{2}{3} a_{2}+c
$$

The stacking vector $\boldsymbol{t}_{1}$ shifts the positions like $\mathrm{A}_{\uparrow} \rightarrow \mathrm{A}_{\downarrow}^{\prime}, \mathrm{B}_{\uparrow} \rightarrow \mathrm{B}_{\downarrow}^{\prime}, \mathrm{C}_{\uparrow} \rightarrow \mathrm{C}_{\downarrow}^{\prime}$, while $\boldsymbol{t}_{2}$ and $t_{2}^{\prime}$ shift the positions like $\mathrm{A}_{\uparrow} \rightarrow \mathrm{B}_{\downarrow}, \mathrm{B}_{\uparrow} \rightarrow \mathrm{C}_{\downarrow}, \mathrm{C}_{\uparrow} \rightarrow \mathrm{A}_{\downarrow}$, and $\mathrm{A}_{\uparrow}^{\prime} \rightarrow \mathrm{C}_{\downarrow}^{\prime}, \mathrm{B}_{\uparrow}^{\prime} \rightarrow \mathrm{A}_{\downarrow}^{\prime}, \mathrm{C}_{\uparrow}^{\prime} \rightarrow \mathrm{B}_{\downarrow}^{\prime}$, respectively. An infinite number of periodic and non-periodic polytypes can be obtained by a random occurrence of the stacking vectors $\boldsymbol{t}_{i}$ and $\boldsymbol{t}_{i}{ }_{i}\left(i=1\right.$ and 2) similarly for $R_{2} T_{7}$ formed based on the stacking of the structural block consisting of a sub-block layer of the $R T_{3}$ stoichiometry (formed with one $R_{2} T_{4}$ unit layer and one $R T_{5}$ unit layer) and a sub-block layer of the $R_{5} T_{19}$ stoichiometry (formed with one $R_{2} T_{4}$ unit layer and three $R T_{5}$ unit layers).

A similar argument holds true in deducing the MDO polytypes. In the case of the OD groupoid family for the $R_{2} T_{7}$ phase with the new crystal structure, one generating operation is the $\sigma$-PO of $6_{6}$, indicated in Fig. 11(d). Continuous application of this operation yields a MDO polytype (MDO1) with the $\mathrm{A}_{\uparrow} \mathrm{A}_{\downarrow}^{\prime}$ stacking sequence. The unit cell of the MDO1 polytype 


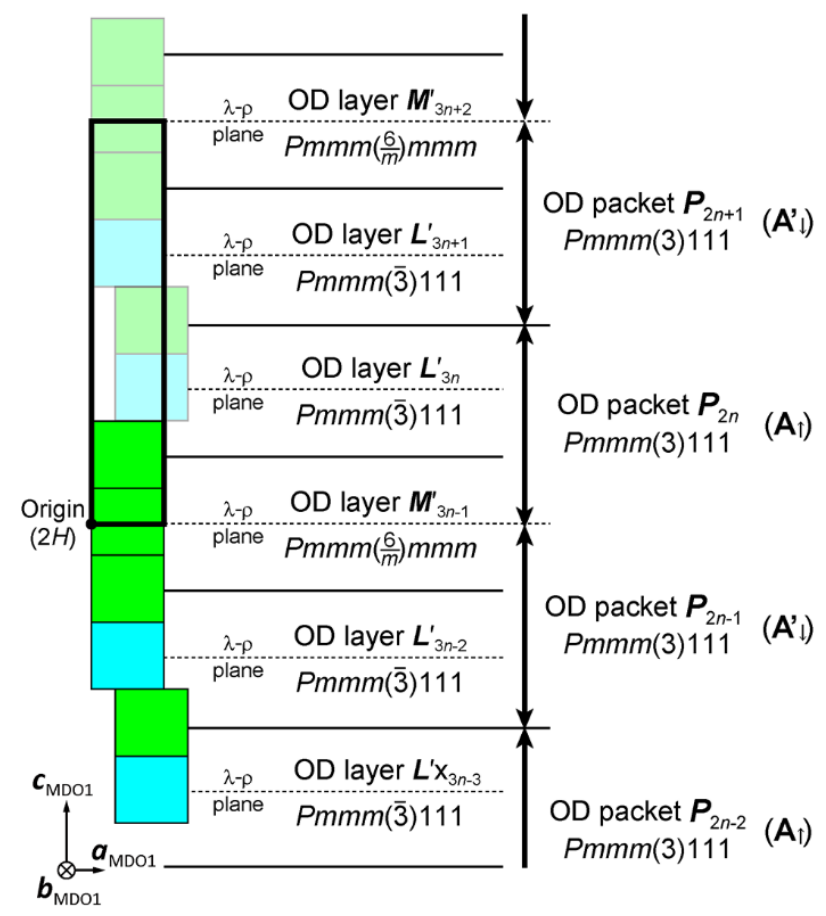

Figure 12. Schematic illustration of the (imaginary) crystal structure of the intermetallic compound $(\mathrm{Nd}, \mathrm{Mg})_{2}(\mathrm{Ni}, \mathrm{Al})_{7}(2 \mathrm{H}$-polytype). The constituting OD layers and packets are described in the right-hand of the figure.

belongs to the space group of $P \overline{6} m 2$ with a hexagonal unit cell of $\boldsymbol{a}_{1}^{(M D O 1)}=\boldsymbol{a}_{1}, \boldsymbol{a}_{2}^{(M D O 1)}=\boldsymbol{a}_{2}$ and $\boldsymbol{c}^{(\mathrm{MDO} 1)}=2 \boldsymbol{c}_{0}$ because the $\sigma$-PO of $6_{6}$ becomes a total operation, $\overline{6}^{+}$, in the hexagonal cell (Fig. 12). In the Ramsdell notation, the MDO1 polytype is designated $2 H$. Another generating operation is the $\sigma-\mathrm{PO}$ of $\overline{3}$ indicated in Fig. 11(e). Continuous application of this operation results in another MDO polytype (MDO2) with the $A_{\uparrow} B_{\downarrow} C_{\uparrow} A_{\downarrow} B_{\uparrow} C_{\downarrow}$ stacking sequence (Fig. 8).

The MDO2 polytype belongs to the space group of $R \overline{3} m$ with the rhombohedral unit cell of $\boldsymbol{a}_{1}^{(M D O 2)}=\boldsymbol{a}_{1}, \boldsymbol{a}_{2}^{(M D O 2)}=\boldsymbol{a}_{2}$, and $\boldsymbol{c}^{(\mathrm{MDO} 2)}=6 \boldsymbol{c}_{0}$ because the $\sigma$-PO of $\overline{3}$ becomes a total operation $g(1 / 6,1 / 3,1 / 3)$ in the rhombohedral cell. In the Ramsdell notation, the MDO2 polytype is designated $6 R$. The experimentally observed polytype is $R \overline{3} m$ (MDO2), which is the second simplest polytype in the OD family. Of importance to note here is that the actual number of 
block layers in the unit cell for the $6 R$ polytype is six $\left(\mathrm{A}_{\uparrow} \mathrm{B}_{\downarrow} \mathrm{C}_{\uparrow} \mathrm{A}_{\downarrow} \mathrm{B}_{\uparrow} \mathrm{C}_{\downarrow}\right)$ but not three (ABC), if we take into account of the inversion symmetry between two neighboring block layers on their stacking.

\subsection{Stacking faults and electron diffraction}

Diffraction from OD structures usually produces two different types of reflections; family reflections and characteristic reflections [20, 34]. Family reflections appear at the common positions for all polytypes if they belong to the same OD family, while characteristic reflections appear at different positions specific to a given polytype, with which polytype identification is possible. If some different polytypes are incorporated or if some stacking disorder is present, sharp streaks are observed in the reciprocal lattice rows of characteristic reflections while discrete diffraction spots are preserved for family reflections, as far as the stacking made is only those allowed to form the same OD family $[21,35]$. On the other hand, if some different phases are incorporated in the form of intergrowth or if some stacking disorder that is not allowed to form the same OD family occurs, streaks are observed in the reciprocal lattice rows of both family and characteristic reflections.

The reflection conditions for the family reflections can be obtained by considering reflection conditions for the so-called 'family structure', a fictitious superimposed structure that is obtained by simultaneous application of all possible $\sigma$-POs $[20,21,35]$. The reflection conditions for the family reflections of $R_{2} T_{7}$ with the new crystal structure are described as follows:

$$
0 H \bar{H} L: H=3 m, L=3 n(m, n: \text { integer })
$$

where the indices in capital letters refer to the unit cell of the MDO2 $(6 R)$ polytype. Indexing of 
the SAED patterns of Fig. 3(b) was made assuming the MDO2 (6R) polytype. Calculation of SAED patterns with the $[2 \overline{1} \overline{1} 0]$ incidence indicates that the $m[01 \overline{1} L]^{*}$ reciprocal lattice rows $(m$ $=3 n$, where $m$ and $n$ are integers) correspond to those of family reflections, while the $m[01 \overline{1} L]^{*}$ reciprocal lattice rows $(m \neq 3 n)$ to those of characteristic reflections. Although the intensity is fairly low, streaks are observed in Fig. 3(b) along reciprocal lattice rows of both family and characteristic reflections. This indicates either the incorporation of some different phases in the form of intergrowth or the introduction of some stacking disorder that is not allowed to form the same OD family. When judged from the HAADF-STEM image of Fig. 4, the intergrowth structures, in which, for example, a sub-block layer of either $R T_{3}$ or $R_{5} T_{19}$ stoichiometry is missed (SF's 1 and 2 in Fig. 4), are identified to be responsible for the occurrence of streaks. In other words, $R_{2} T_{7}$ with the new crystal structure is relatively stable with respect to other polytype, since the incorporation of other polytypes is less frequent.

4.4. Further possible crystal structures with different stoichiometric compositions and with different stacking structures

A series of compounds in the $R-T$ system, $R T_{3}, R_{2} T_{7}$ and $R_{5} T_{19}$ possess a crystal structure based on the stacking of block layers, each of which has their own stoichiometric composition consisting of one unit layer of the $R_{2} T_{4}$ (Laves)-type and some unit layers of the $R T_{5}$-type [12-14, 36] (Fig. 1). As the number ( $n$ ) of $R T_{5}$-type unit layers in the block layer increases, intermetallics richer in $T$ ( $n=1,2$ and 3 for $R T_{3}, R_{2} T_{7}$ and $R_{5} T_{19}$, respectively) are successively formed so as to be formulated as $R T_{x}$ with $x=(5 n+4) /(n+2)$ [12]. Theoretically, it is possible to form intermetallic compounds $R T_{x}$ with the value of $n$ exceeding $4\left(R T_{4}, R_{7} T_{29}\right.$ and $R_{4} T_{17}$ for, $n=4,5$ and 6 , respectively). Although these intermetallics with higher $x$ values have never been reported in the 
$R-T$ binary system, the intermetallic compound $R T_{4}\left(\mathrm{La}_{5} \mathrm{MgNi}_{24}\right)$ with $n=4$ has indeed been reported to form in the La-Mg-Ni ternary system [37]. The intermetallic compound $R T_{4}$ is reported to exhibit better discharge capacity than that based on $R_{5} T_{19}$ when examined as a negative electrode of Ni-MH batteries [37].

The new crystal structure of $R_{2} T_{7}$ presently identified is different from those of these intermetallics $R T_{x}$ with higher $x$ values in that although the structure is formed similarly based on the stacking of block layers, the block layer for the new crystal structure consists of one subblock layer of the $R T_{3}$ stoichiometry (formed with one $R_{2} T_{4}$ unit layer and one $R T_{5}$ unit layer) and one sub-block layer of the $R_{5} T_{19}$ stoichiometry (formed with one $R_{2} T_{4}$ unit layer and three $R T_{5}$ unit layers), which alternately stack on top of each other. If the block layer is allowed to consist of more than two kinds of sub-block layers (either of $R T_{3}, R_{2} T_{7}$ or $R_{5} T_{19}$ stoichiometry), it is possible to form an infinite number of intermetallic compounds at stoichiometric compositions in between $R T_{2}$ and $R T_{5}$. Although the stoichiometric composition of the new structure formed with one sub-block layer of the $R T_{3}$ stoichiometry and one sub-block layer of the $R_{5} T_{19}$ stoichiometry is $R_{2} T_{7}$, the stoichiometric composition is changed to $R_{7} T_{23}$ if the sub-block layer of the $R_{5} T_{19}$ stoichiometry is replaced with that of the $R_{2} T_{7}$ stoichiometry (Fig. 13(b)). This crystal structure of $R_{7} T_{23}\left(\mathrm{La}_{5} \mathrm{Mg}_{2} \mathrm{Ni}_{23}\right)$ has indeed been observed to exist in the $\mathrm{La}-\mathrm{Mg}-\mathrm{Ni}$ ternary system $[1,38]$. The intermetallic compound $R_{7} T_{23}$ is reported also to exhibit better discharge capacity than that based on $R T_{5}$ [1]. Similarly, the intermetallic compound $R_{3} T_{11}$ is formed with the block layer consisting of one $R_{2} T_{7}$ sub-block layer and one $R_{5} T_{19}$ sub-block layer (Fig. 13(c)). Another crystal structure of the intermetallic compound $R_{2} T_{7}$ is formed with the block layer consisting of one $R T_{3}$ sub-block layer, one $R_{2} T_{7}$ sub-block layer and one $R_{5} T_{19}$ subblock layer (Fig. 13(d)). If the block layer consists of two $R T_{3}$ sub-block layers and either $R_{2} T_{7}$ or 


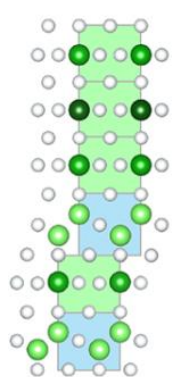

(a) $R_{2} T_{7}$

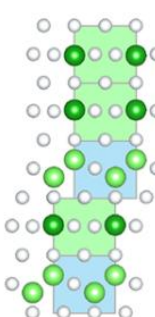

(b) $R_{7} T_{23}$

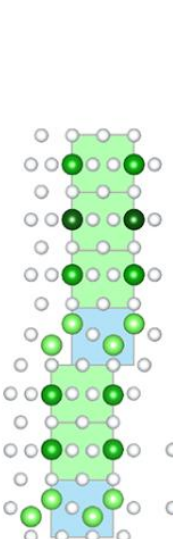

(c) $R_{3} T_{11}$

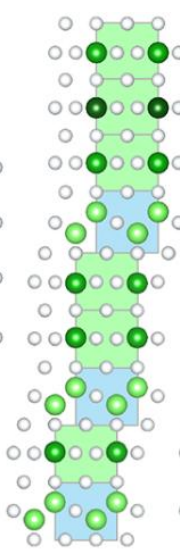

(d) $R_{2} T_{7}$

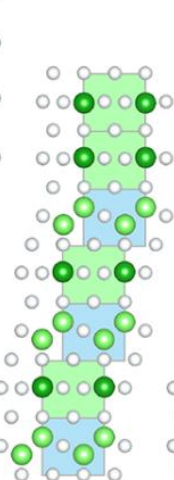

(e) $R_{5} T_{16}$

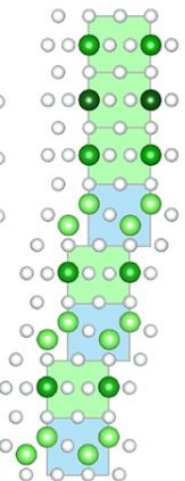

(f) $R_{11} T_{37}$

Figure 13. Further possible crystal structures with different stoichiometric compositions and with different stacking structures.

$R_{5} T_{19}$ sub-block layer, the stoichiometric composition is either $R_{5} T_{16}$ (Fig. 13(e)) or $R_{11} T_{37}$ (Fig. 13(f)). Needless to say, all these crystal structures can be described with the OD theory (as described in Sections 4.1 and 4.2), as far as they are based on the stacking of block layers.

The crystal structure variation as mentioned above is empirically known not to occur in simple $R$ - $T$ systems, unless $\mathrm{Mg}$ is added to replace some $R$ atoms [1,37-41]. In this sense, $\mathrm{Mg}$ is considered to play an important role in controlling the stability of crystal structures. Electrochemical properties of intermetallic compounds $R T_{x}$ containing $\mathrm{Mg}$ have frequently been reported to be better than those of their base compounds [3, 7, 8, 11, 42-46]. Our preliminary experiment has indicated that the alloy 2 containing grains of the $R_{2} T_{7}$ phase with the new crystal structure tends to exhibit better discharge capacity than the alloy 1 containing grains of the $R_{2} T_{7}$ phase with the conventional $2 H$ polytype structure (See the Supplementary Figure S1). Their properties cannot simply be inferred from the rule of mixture of the intermetallic compounds $R_{2} T_{4}$ and $R T_{5}$ that constitute the block layer $[11,42,45]$. This indicates that there is a chance to find out a new compound that exhibits excellent electrochemical properties as a negative electrode of Ni-MH batteries through controlling crystal structures based on the stacking of 
block layers with additions of $\mathrm{Mg}$ and some other alloying elements. $\mathrm{Mg}$ is reported to preferentially occupy $R$ atom sites in the $R_{2} T_{4}$ unit layer of the block layer in a series of compounds $R T_{x}$ in the $R-T$ system. In the presence of a quaternary element that has preferential occupancy in particular sites in either $R_{2} T_{4}$ or $R T_{5}$ unit layer, there is a possibility that the occupancy behavior of $\mathrm{Mg}$ in the $R_{2} T_{4}$ unit layer varies with sub-block layers if the block layer consists of sub-block layers with different stoichiometric compositions, as in the presently identified new crystal structure of $R_{2} T_{7}$. We believe that $\mathrm{Al}$ is one of the effective quaternary elements that also play an important role, since $\mathrm{Al}$ has been known to preferentially occupy $T$ sites between $R_{2} T_{4}$ and $R T_{5}$ unit layers and between $R T_{5}$ unit layers [37]. Indeed, the present study clearly indicates that crystal grains of the $R_{2} T_{7}$ phase with the new crystal structure tend to possess more $\mathrm{Mg}$ and $\mathrm{Al}$ than those of the $R_{2} T_{7}$ phase with the conventional $2 \mathrm{H}$ polytype structure. Ways to control the constitution and stacking of block layers of the intermetallic compounds $R T_{x}$ correlating with the occupancy behavior of $\mathrm{Mg}$ and other alloying elements are currently under survey experimentally and theoretically in our research group.

\section{CONCLUSIONS}

(1) The new crystal structure of the intermetallic compound $(\mathrm{Nd}, \mathrm{Mg})_{2}(\mathrm{Ni}, \mathrm{Al})_{7}$ has been determined as that based on the stacking of the block layer of the $R_{2} T_{7}$ stoichiometry that consists of a sub-block layer of the $R T_{3}$ stoichiometry (formed with one $R_{2} T_{4}$ unit layer and one $R T_{5}$ unit layer) and a sub-block layer of the $R_{5} T_{19}$ stoichiometry (formed with one $R_{2} T_{4}$ unit layer and three $R T_{5}$ unit layers), which alternately stack on top of each other in the stacking of the ABCtype. This is quite different from the crystal structures (either $2 H$ or $3 R$ polytypes) usually reported for the intermetallic compound $R_{2} T_{7}$, in which the block layer of the $R_{2} T_{7}$ stoichiometry 
consists simply of one $R_{2} T_{4}$ unit layer and two $R T_{5}$ unit layers.

(2) The new crystal structure is described based on the order-disorder theory. The crystal structure belongs to the category IV OD structure composed of two types of non-polar OD layers $\left(L^{\prime}\right.$ and $M^{\prime}$ ). Due to the selectivity property of the orientation of the OD layer $L^{\prime}$, there are two possible equivalent positions to stack an OD layer $L^{\prime}$ on top of an OD layer $L^{\prime}$ or $M^{\prime}$. The OD groupoid family symbol expressing the OD structure is:

$$
\begin{gathered}
\operatorname{Pmmm}\left(\frac{6}{m}\right) m m m \operatorname{Pmmm}(\overline{3}) 111 \operatorname{Pmmm}(\overline{3}) 111 \\
{[2 / 3,1 / 3] \quad[2 / 3,1 / 3]}
\end{gathered}
$$

(3) The space groups for the two simplest forms (MDO polytypes) of the intermetallic compound $R_{2} T_{7}$ are determined to be $P \overline{6} m 2$ (MDO1) and $R \overline{3} m$ (MDO2). In the Ramsdell notation, these forms are designated $2 H$ and $6 R$, respectively. The experimentally observed polytype is $R \overline{3} m$ (MDO2), which is the second simplest polytype in the OD family.

\section{ACKNOWLEDGMENT}

This work was supported by JSPS KAKENHI grant numbers 24246113 and 25709066, and the Elements Strategy Initiative for Structural Materials (ESISM) from the Ministry of Education, Culture, Sports, Science and Technology (MEXT) of Japan, and in part by Advanced Low Carbon Technology Research and Development Program (ALCA) from the Japan Science and Technology Agency (JST). 


\section{REFERENCES}

[1] Kohno T, Yoshida H, Kawashima F, Inaba T, Sakai I, Yamamoto M, et al. Hydrogen storage properties of new ternary system alloys: $\mathrm{La}_{2} \mathrm{MgNi}_{9}, \mathrm{La}_{5} \mathrm{Mg}_{2} \mathrm{Ni}_{23}, \mathrm{La}_{3} \mathrm{MgNi}_{14}$. $\mathrm{J}$ Alloys Compd. 2000;311:L5-L7.

[2] Akiba E, Hayakawa H, Kohno T. Crystal structures of novel La-Mg-Ni hydrogen absorbing alloys. J Alloys Compd. 2006;408:280-3.

[3] Zhang FL, Luo YC, Wang DH, Yan RX, Kang L, Chen JH. Structure and electrochemical properties of $\mathrm{La}_{2-x} \mathrm{Mg}_{x} \mathrm{Ni}_{7.0}(x=0.3-0.6)$ hydrogen storage alloys. J Alloys Compd. 2007;439:1818.

[4] Denys RV, Riabov AB, Yartys VA, Sato M, Delaplane RG. Mg substitution effect on the hydrogenation behaviour, thermodynamic and structural properties of the $\mathrm{La}_{2} \mathrm{Ni}_{7}-\mathrm{H}(\mathrm{D})_{2}$ system. $\mathrm{J}$ Solid State Chem. 2008;181:812-21.

[5] Yartys VA, Vajeeston P, Riabov AB, Ravindran P, Denys RV, Maehlen JP, et al. Crystal chemistry and metal-hydrogen bonding in anisotropic and interstitial hydrides of intermetallics of rare earth $(R)$ and transition metals $(T), R T 3$ and $R_{2} T_{7}$. Z Kristallogr. 2008;223:674-89.

[6] Crivello JC, Zhang J, Latroche M. Structural Stability of $\mathrm{AB}_{\mathrm{y}}$ Phases in the (La,Mg)-Ni System Obtained by Density Functional Theory Calculations. J Phys Chem C. 2011;115:25470-8. [7] Liu YF, Cao YH, Huang L, Gao MX, Pan HG. Rare earth-Mg-Ni-based hydrogen storage alloys as negative electrode materials for Ni/MH batteries. J Alloys Compd. 2011;509:675-86.

[8] Zhang QG, Zhao B, Fang MH, Liu CR, Hu QM, Fang F, et al. $\left(\mathrm{Nd}_{1.5} \mathrm{Mg}_{0.5}\right) \mathrm{Ni}_{7}-\mathrm{Based}$ Compounds: Structural and Hydrogen Storage Properties. Inorg Chem. 2012;51:2976-83.

[9] Serin V, Zhang JX, Magen C, Serra R, Hytch MJ, Lemort L, et al. Identification of the atomic scale structure of the $\mathrm{La}_{0.65} \mathrm{Nd}_{0.15} \mathrm{Mg}_{0.20} \mathrm{Ni}_{3.5}$ synthesized by spark plasma sintering alloy. Intermetallics. 2013;32:103-8.

[10] Yasuoka S, Magari Y, Murata T, Tanaka T, Ishida J, Nakamura H, et al. Development of high-capacity nickel-metal hydride batteries using superlattice hydrogen-absorbing alloys. J Power Sources. 2006;156:662-6.

[11] Oesterreicher H, Clinton J, Bittner H. Hydrides of La-Ni Compounds. Mater Res Bull. 1976;11:1241-7.

[12] Khan Y. Constitution of $R-T$ Systems ( $R=$ Rare Earths and Yttrium, $T=$ Iron, Cobalt and Nickel). Z Metallkd. 1974;65:489-95.

[13] Takeda S, Kitano Y, Komura Y. Polytypes of the Intermetallic Compound $\mathrm{Sm}_{5} \mathrm{Ni}_{19}$. J LessCommon Met. 1982;84:317-25.

[14] Takeda S, Horikoshi H, Komura Y. Microstructure of $\mathrm{Sm}_{5} \mathrm{Ni}_{19}$ Intermetallic Compound Observed by High-Resolution Electron-Microscope. Journal of Microscopy-Oxford. 1983;129:347-58.

[15] Ramsdell LS. Studies on Silicon Carbide. Am Mineral. 1947;32:64-82.

[16] Dornberger-Schiff K. On Order-Disorder Structures (OD-Structures). Acta Crystallogr. 1956;9:593-601.

[17] Dornberger-Schiff K. On the Nomenclature of the 80 Plane Groups in 3 Dimensions. Acta Crystallogr. 1959;12:173-.

[18] Dornberger-Schiff, K. Grundzüge einer Theorie der OD-Strukturen aus Schichten. Abh Dtsch Akad Wiss. 1964;3:1-107.

[19] Dornberger-Schiff K. OD Structures, - a Game and a Bit More. Krist Techn. 1979;14:1027-45.

[20] Durovič S. Fundamentals of the OD theory. In: Merlino S, editor. Modular Aspects of 
Minerals. Budapest, Hungary: Eőtvős University Press; 1997. p. 3-28.

[21] Ferraris G, Makovicky E, Merlino S. Crystallography of Modular Materials. Oxford, UK: Oxford University Press; 2008.

[22] Jeffery JW. Unusual X-Ray Diffraction Effects from a Crystal of Wollastonite. Acta Crystallogr. 1953;6:821-5.

[23] Dornbergerschiff K, Liebau F, Thilo E. Zur Struktur Des Beta-Wollastonits, Des Maddrellschen Salzes Und Des Natriumpolyarsenats. Acta Crystallogr. 1955;8:752-4.

[24] Yokobayashi H, Kishida K, Inui H, Yamasaki M, Kawamura Y. Enrichment of Gd and Al atoms in the quadruple close packed planes and their in-plane long-range ordering in the long period stacking-ordered phase in the Mg-Al-Gd system. Acta Mater. 2011;59:7287-99.

[25] Kishida K, Yokobayashi H, Inui H, Yamasaki M, Kawamura Y. The crystal structure of the LPSO phase of the 14H-type in the Mg-Al-Gd alloy system. Intermetallics. 2012;31:55-64.

[26] Kishida K, Yokobayashi H, Inui H. The most stable crystal structure and the formation processes of an order-disorder (OD) intermetallic phase in the Mg-Al-Gd ternary system. Philos Mag. 2013;93:2826-46.

[27] Okamoto NL, Yasuhara A, Inui H. Order-Disorder Structure of the $\delta_{1 \mathrm{k}}$ Phase in the Fe-Zn System Determined by Scanning Transmission Electron Microscopy. Acta Mater. 2014;81:34557.

[28] Kopsky V, Litvin DB. International Tables for Crystallography, Vol. E: Superperiodic group. 2nd ed. Chichester, UK: John Wiley \& Sons, Ltd; 2010.

[29] Dornbergerschiff K, Grell H. Geometrical Properties of MDO Polytypes and Procedures for Their Derivation: OD Families Containing OD Layers of M Greater-Than 1 Kinds and Their MDO Polytypes. Acta Crystallogr A. 1982;38:491-8.

[30] Grell H, Dornbergerschiff K. Symbols for OD Groupoid Families Referring to OD Structures (Polytypes) Consisting of More Than One Kind of Layer. Acta Crystallogr A. 1982;38:49-54.

[31] Merlino S, Orlandi P, Perchiazzi N, Basso R, Palenzona A. Polytypism in Stibivanite. Can Mineral. 1989;27:625-32.

[32] Durovic S. Notion of Packets in Theory of OD Structures of M Greater-Than 1 Kinds of Layers - Examples - Kaolinites and $\mathrm{MoS}_{2}$. Acta Crystallogr B. 1974;30:76-8.

[33] Hybler J, Durovic S. The OD interpretation of the crystal structure of kettnerite $\mathrm{CaBiOFCO}_{3}$. Acta Crystallogr A. 2009;65:501-11.

[34] Durovic S, Hybler J. OD structures in crystallography - basic concepts and suggestions for practice. Z Kristallogr. 2006;221:63-76.

[35] Merlino S. OD approach in ninerals: examples and applications. In: Merlino S, editor. Modular Aspects of Minerals. Budapest, Hungary: Eőtvős University Press; 1997. p. 29-54.

[36] Iwase K, Mori K. Crystal structure and hydrogen storage property of $\mathrm{Nd}_{2} \mathrm{Ni}_{7}$ superlattice alloy. Int J Hydrogen Energy. 2013;38:5316-21.

[37] Ozaki T, Kanemoto M, Kakeya T, Kitano Y, Kuzuhara M, Watada M, et al. Stacking structures and electrode performances of rare earth-Mg-Ni-based alloys for advanced nickelmetal hydride battery. J Alloys Compd. 2007;446:620-4.

[38] Kohno T, Takeno S, Yoshida H, Kanda M. Structural analysis of La-Mg-Ni-based new hydrogen storage alloy. Res Chem Intermed. 2006;32:437-45.

[39] Yamamoto T, Inui H, Yamaguchi M, Sato K, Fujitani S, Yonezu I, et al. Microstructures and hydrogen absorption/desorption properties of La-Ni alloys in the composition range of La77.8 83.2 at.\%Ni. Acta Mater. 1997;45:5213-21. 
[40] Inui H, Yamamoto T, Di Z, Yamaguchi M. Microstructures and defect structures in intermetallic compounds in the La-Ni alloy system. J Alloys Compd. 1999;293:140-5.

[41] Di Z, Yamamoto T, Inui H, Yamaguchi M. Characterization of stacking faults on basal planes in intermetallic compounds $\mathrm{La}_{5} \mathrm{Ni}_{19}$ and $\mathrm{La}_{2} \mathrm{Ni}_{7}$. Intermetallics. 2000;8:391-7.

[42] Oesterreicher H, Bittner H. Hydride Formation in $\mathrm{La}_{1-x} \mathrm{Mg}_{x} \mathrm{Ni}_{2}$. J Less-Common Met. 1980;73:339-44.

[43] Kadir K, Kuriyama N, Sakai T, Uehara I, Eriksson L. Structural investigation and hydrogen capacity of $\mathrm{CaMg}_{2} \mathrm{Ni}_{9}$ : a new phase in the $\mathrm{AB}_{2} \mathrm{C}_{9}$ system isostructural with $\mathrm{LaMg}_{2} \mathrm{Ni}_{9}$. $\mathrm{J}$ Alloys Compd. 1999;284:145-54.

[44] Tang R, Liu YN, Zhu CC, Zhu JW, Yu G. Effect of Mg on the hydrogen storage characteristics of $\mathrm{MI}_{1-x} \mathrm{Mg}_{x} \mathrm{Ni}_{2.4} \mathrm{Co}_{0.6}$ ( $x=0$-0.6) alloys. Mater Chem Phys. 2006;95:130-4.

[45] Terashita N, Akiba E. Hydriding properties of $\left(\mathrm{Mg}_{1-x} \mathrm{M}_{x}\right) \mathrm{Ni}_{2}$ C15-type laves phase alloys. Mater Trans. 2006;47:1890-3.

[46] Dong XP, Lu FX, Yang LY, Zhang YH, Wang XL. Influence of spark plasma sintering temperature on electrochemical performance of $\mathrm{La}_{0.80} \mathrm{Mg}_{0.20} \mathrm{Ni}_{3.75}$ alloy. Mater Chem Phys. 2008;112:596-602. 
Figure 1.Crystal structures of (a) $R T_{2}\left(R_{2} T_{4}\right)$, (b) $R T_{3}$, (c) $R_{2} T_{7}$, (d) $R_{5} T_{19}$ and (e) $R T_{5} . R T_{2}$ and $R T_{5}$ have $\mathrm{C} 15$ (Laves)- and $\mathrm{CaCu}_{5}$-type structures, respectively. The crystal structures of (b) $R T_{3}$, (c) $R_{2} T_{7}$ and (d) $R_{5} T_{19}$ consist of block layers, each of which consists of $R_{2} T_{4}$ and $R T_{5}$ unit layers, and the polytype $2 H$ is illustrated for these intermetallics.

Figure 2. (a) Typical high-resolution HAADF-STEM image and (b) SAED pattern with the [2 $1 \overline{1}$ 0 ] incidence for a crystal grain of the $R_{2} T_{7}$ phase in the alloy 1 . Positions of sub-block layers of the $R T_{5}$ - and $R_{2} T_{4}$-types are indicated in the figure.

Figure 3. (a) Typical high-resolution HAADF-STEM image and (b) SAED pattern with the $[2 \overline{1} \overline{1}$ 0] incidence for a crystal grain of the $R_{2} T_{7}$ phase in the alloy 2. Positions of sub-block layers of the $R T_{3}$ - and $R_{5} T_{19}$-types are indicated in the figure.

Figure 4. Low-magnification HAADF-STEM image of the $R_{2} T_{7}$ phase in the alloy 1. Positions of stacking faults (SF1 and SF2) are indicated in the right of the figure.

Figure 5. Crystal structures of (a) $2 H-R T_{3}$, (b) $2 H-R_{2} T_{7}$, and (c) $2 H-R_{5} T_{19}$ projected along the [2 110 ] direction. The constituting OD layers and packets are described in the righthand of each figure.

Figure 6. (a,c,d,e) [2 $\begin{array}{lll}\overline{1} & 1 & 0]\end{array}$ and (b,f) [0001] projections of the OD layers (a,b) $L$ and (c,d,e) $M$, respectively, in the (c) $R T_{3}$, (d) $R_{2} T_{7}$, and (e) $R_{5} T_{19}$ phases. (g,h) Diagrams of symmetry elements for the $p \overline{3} \mathrm{~m} 1$ and $p 6 / \mathrm{mmm}$ layer groups corresponding to the $\lambda$ POs in the OD layers $L$ and $M$, respectively.

Figure 7. (a) [2 110$]$ and (b) [0001] projections of the OD packet in the $R_{2} T_{7}$ phase. (c) Arrays of symbolic figures showing the symmetry of the OD packet. (d,e) Two different manners of stacking the adjacent OD packet on the preceding OD packet at the position $\mathrm{A}$. (d) $\mathrm{A} \Rightarrow \mathrm{A}$ ' with the stacking vector $\boldsymbol{t}_{1}$, (e) $\mathrm{A} \Rightarrow \mathrm{B}$ with the stacking vector $\boldsymbol{t}_{2}$. (f) One of the two manners of stacking the adjacent OD packet on the preceding OD packet at the position $A^{\prime}\left(A^{\prime} \Rightarrow C^{\prime}\right)$.

Figure 8. Schematic illustration of the crystal structure of the intermetallic compound $(\mathrm{Nd}, \mathrm{Mg})_{2}(\mathrm{Ni}, \mathrm{Al})_{7}(6 R$-polytype). The constituting OD layers and packets are described in the right-hand of the figure.

Figure 9. (a,c) [2 $1 \overline{1} 10]$ and (b,d) [0001] projections of the OD layers (a,b) $L^{\prime}$ and (c,d) $M^{\prime}$, respectively, in the $(\mathrm{Nd}, \mathrm{Mg})_{2}(\mathrm{Ni}, \mathrm{Al})_{7}$ phase of the new crystal structure.

Figure 10. Schematic illustration of the OD packets $P_{2 n}$ and $P_{2 n+1}$ constituting the $(\mathrm{Nd}, \mathrm{Mg})_{2}(\mathrm{Ni}, \mathrm{Al})_{7}$ phase of the new crystal structure.

Figure 11. (a) [2 $\left.\begin{array}{lll}2 & 1 & 0\end{array}\right]$ and (b) [0001] projections of the $\mathrm{OD}$ packet in the $(\mathrm{Nd}, \mathrm{Mg})_{2}(\mathrm{Ni}, \mathrm{Al})_{7}$ phase of the new crystal structure. (c) Arrays of symbolic figures showing the symmetry of the OD packet. $(\mathrm{d}, \mathrm{e})$ Two different manners of stacking the adjacent OD packet on the preceding OD packet at the position $A_{\uparrow}$. (d) $A_{\uparrow} \rightarrow A_{\downarrow}^{\prime}$ with the stacking vector $t_{1}$, (e) $A_{\uparrow} \rightarrow B_{\downarrow}$ with the stacking vector $t_{2}$. (f) One of the two manners of stacking the adjacent OD packet on the preceding OD packet at the position $\mathrm{A}_{\uparrow}^{\prime}\left(\mathrm{A}_{\uparrow}^{\prime} \rightarrow \mathrm{C}_{\downarrow}^{\prime}\right)$.

Figure 12. Schematic illustration of the (imaginary) crystal structure of the intermetallic compound $(\mathrm{Nd}, \mathrm{Mg})_{2}(\mathrm{Ni}, \mathrm{Al})_{7}(2 \mathrm{H}$-polytype). The constituting OD layers and packets are described in the right-hand of the figure. 
Figure 13. Further possible crystal structures with different stoichiometric compositions and with different stacking structures.

Table 1. Nominal compositions of the alloys 1 and 2. 

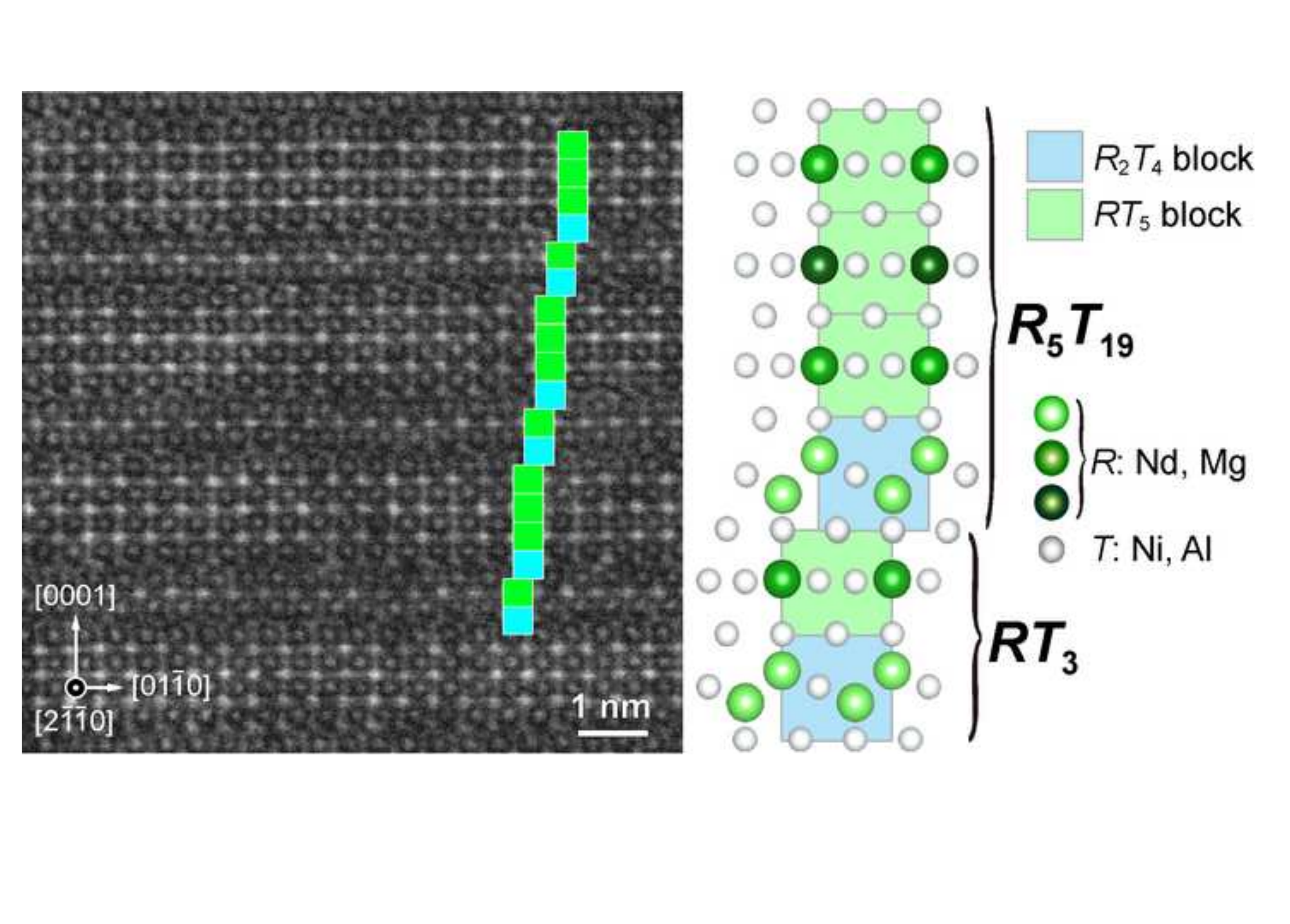

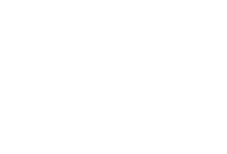

$$
\text { (2) }
$$

更

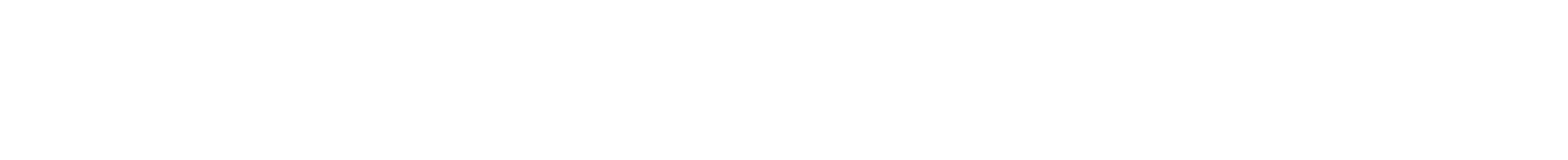

.

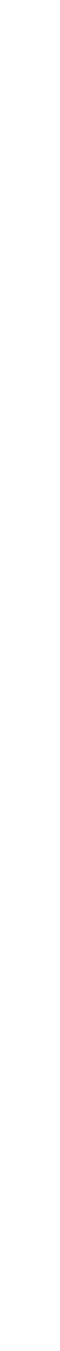

